\title{
An uncoupled thermoelasticity problem for a semi-infinite layer with regard to its proper weight
}

\author{
A. Fesenko, N. Vaysfel'd \\ Faculty of Mathematics, Physics and Information Technologies, Odessa I. I. Mechnikov National University, Ukraine \\ 81anna81@gmail.com,vaysfeld@onu.edu.ua
}

\begin{abstract}
The exact solution of the uncoupled thermoelasticity problem for a semi-infinite elastic layer with regard to its proper weight was constructed. The originality of the proposed paper is based on reducing Lame equations to two jointly and one separately, solvable equations. It allows the application of integral transformations directly to the transformed equations of equilibrium and makes it possible to reduce the initial problem to a one-dimensional vector boundary problem. A special technique is given to calculate multiple integrals containing oscillating functions that appear during the inversion of the transforms. The character of the temperature and proper weight influence on the value of normal stress on the lateral face of the semi-infinite layer, the zone of tensile stress depending on the shapes of the distributed load section and the temperature and Poisson's ratio is established. The parameters of dimensionless mechanical load and temperature, when the separation of the side wall of the semi-infinite layer can be eliminated, were established. A study of the influence of the layer's proper weight on the stress emerging on the layer's edge is conducted. The constructed exact solution can be used as a model for solving a similar class of problems by numerical methods.
\end{abstract}

KEYwORDS. Uncoupled Thermoelasticity Problem; Semi-infinite Layer; Proper Weight; Tensile Stress.

\section{OPEN ACCESS}

Citation: Fesenko, A., Vaysfel'd, N. An uncoupled thermoelasticity problem for a semi-infinite layer with regard of its proper weight, Frattura ed Integrità Strutturale, 48 (2019) 768-792.

Received: 12.12 .2019

Accepted: 14.03 .2019

Published: 01.04.2019

Copyright: (C) 2019 This is an open access article under the terms of the CC-BY 4.0, which permits unrestricted use, distribution, and reproduction in any medium, provided the original author and source are credited.

\section{INTRODUCTION}

\footnotetext{
A number of methods of three-dimensional problem studies in elasticity are based on representations of homogeneous Lame equation solutions using harmonic and biharmonic functions. Such representations were given by J. V. Boussinesq, W. Thomson (Lord Kelvin), and P. G. Tait [1] where the possibility of reducing the number of harmonic functions to three was also considered. B. G. Galerkin presented the general solution of homogeneous equilibrium equations for an isotropic body through three biharmonic functions. In the works of P. F. Papkovich [2] and H. Neuber [3] a solution form containing four harmonic functions reducing the Lame equations to a harmonic sequence with unshared boundary conditions was proposed.
} 
Using functions of a complex variable and integrals of Cauchy type in monographs G.V. Kolosov [4] and I. I. Muskhelishvili [5] developed an effective method for solving plane boundary problems of elasticity, which later became classical. Although this method is not directly applicable to the spatial problems of elasticity, the apparatus of functions of a complex variable is also used in solving problems of this class. So, G. N. Polozhii [6] proposed a method for solving axisymmetric spatial problems in elasticity, it involves using two P-analytic functions and is an analog of Kolosov Muskhelishvili's complex potential method.

Another way to investigate three-dimensional problems is the method of integral equations, with its help, the existence and unique theorems for the solution of boundary value problems can be proved. This method often serves as a basis for developing algorithms for the numerical solution of elasticity problems. The monograph [7] is devoted to the method of integral equations.

The method of integral transforms is widely used in spatial problems of elasticity. With the help of the corresponding integral transform, a transition is made to a simpler problem in the domain of transforms. An extensive bibliography of papers on the use of this method in problems of elasticity theory is given in the monograph by Ya. S. Uflyand [8].

Thus, the above methods, using different representations' solutions through auxiliary harmonic and biharmonic functions, which, on one hand, facilitated solving in terms of these functions, but on the other hand it causes difficulties during the calculation of the original functions These difficulties in itself present a complex mathematical problem.

One of the basic methods to construct an exact solution for spatial problems of elasticity is the Fourier method, which is based on the use of curvilinear orthogonal coordinate systems that allow the separation of variables in the threedimensional Laplace equation for static problems. One notes that solutions obtained by the Fourier method are the initial steps to construct solutions for problems of finite bodies bounded by surfaces described by canonical coordinate systems. Using the method of variable separation demands different representations of the equilibrium equations solutions through the stress functions. With the help of such representations, the original problem is reduced to solving differential equations of a simpler structure. Each stress function in these equations is not related to the others, but it is presented in the boundary conditions together with the others. The solution in the form of Papkovich - Neuber is used most often, because it allows the application of classical solutions of the potential theory by solving boundary value problems, represented in the form of series and integrals containing special functions.

Another way to investigate spatial problems is the method of eigenvector functions, which is a vector analog of the Fourier method, this was proposed in [9]. It involves the construction of vector structure eigen functions on the boundary surface of the bodies. With the help of this method, solutions were obtained for complex three-dimensional problems of elasticity.

In paper [10] a method of two-dimensional singular equations for three-dimensional problems of stationary thermal conductivity and thermoelasticity for bodies with cracks was developed, and several problems for a semi-infinite body with cracks were solved.

A new approach proposed by G. Ya. Popov in [11] is used in this paper. The method is based on reducing Lame equations to one independently solvable and two jointly solved equations. Moreover, the boundary conditions are also separated, which greatly simplifies the calculation technique in comparison with traditional methods. By the method of integral transforms applied directly to the transformed equations of equilibrium and the boundary conditions of the initial stated problem, one reduces it to a one-dimensional vector boundary value problem, which is solved exactly. The problem of elasticity for a quarter space was solved with this method in [12].

The elastic layer, as an important and frequently used model object, has been studied by many authors. Thus, in a static formulation, the axisymmetric contact problems for an elastic layer and strip are considered in [13-15], various mixed boundary-value thermoelasticity problems for a half-layer and a half-space in [16-23], layers with thermal loadings were considered in [24] with no static loading, where the conformal mapping method was used, dynamic problems were investigated in [25-27]. The papers [28, 29] are devoted to solving elasticity problems for bodies with their proper weight. In paper [30] the authors investigated the propagation of thermoelastic waves through the layers and analyzed the importance of thermally nonlinear generalized thermoelastic analysis. In [31] the size-dependent quality factor of thermoelastic damping in a microbeam resonator, based on modified strain gradient elasticity was analyzed. The generalized thermoelasticity theory of the Lord-Shulman model was used to derive the equation of coupled thermoelasticity. The paper [32] is devoted to study functionally graded nanodisks under thermoelastic loading based on the strain gradient theory, where the effects of external load at the inner and outer radii on radial displacement as well as stress components were considered. In [33] the influence of measurement errors on the accuracy of the estimated heat flux and mechanical load on laminated functionally graded plate was investigated. Thermo-elasticity problems of functionally graded materials were evaluated in [34]. In [35] an exact solution for thermoelastic deformations of functionally graded thick rectangular plates was derived. The contact problem of two semi-infinite Kirchhoff plates on the 
elastic layer is considered in [36]. The contact stresses concentrations between the plates and the layer was studied, as it can induce destruction of the engineering structure. The advances in the use of thermoelastic stress analysis for fracture mechanics assessment were reviewed in [37]. The development of techniques to determine stress intensity factor was presented, followed by the application of these techniques to fatigue crack growth.

The work [38] investigates the behavior of a Transvers Crack Tensile specimen undergoing fatigue loading, by means of a Thermoelastic Stress Analysis experimental setup. The information on the stress distribution settling near the crack tips and its evolution with crack growth under fatigue is provided.

A review of the above papers shows that the problem of constructing an exact solution for problems of an elastic layer remains open, despite the variety of methods and in particular numerical solutions. The results obtained, based on the exact solution of the spatial problem can be used as the basis for solving problems by approximate numerical methods. This dictates an increasing interest in the development of analytical methods to solve three-dimensional elasticity problems and in particular for a layer as the most important model object.

The purpose of this paper is to establish the changes in the fields of displacements and stress of the semi-infinite elastic layer for the effects of different types of loading, in particular temperature, mechanical loads and proper weight. Earlier in paper [16], the problem of stationary heat conduction and the elasticity problem for a layer were solved separately. It was stated that using this technique makes it is possible to solve the problem of uncoupled thermoelasticity, which was accomplished in this work. Solving this task requires use of the Green function apparatus, moreover, the Green matrix function, which has not been used for the problems of this type. The proposed work consists of a new approach that makes it possible to obtain an exact solution of mixed uncoupled thermoelasticity, which can be also applied to solve analogical problems with much more complicated boundary conditions.

\section{THE STATEMENT OF THE MIXED UNCOUPLED THERMOELASTICITY PROBLEM}

L et's consider the elastic semi-infinite layer (Fig. 1) occupying the area

$0<x<\infty,-\infty<y<\infty, 0<z<b$

$x, y, z$ are the Cartesian's coordinate system

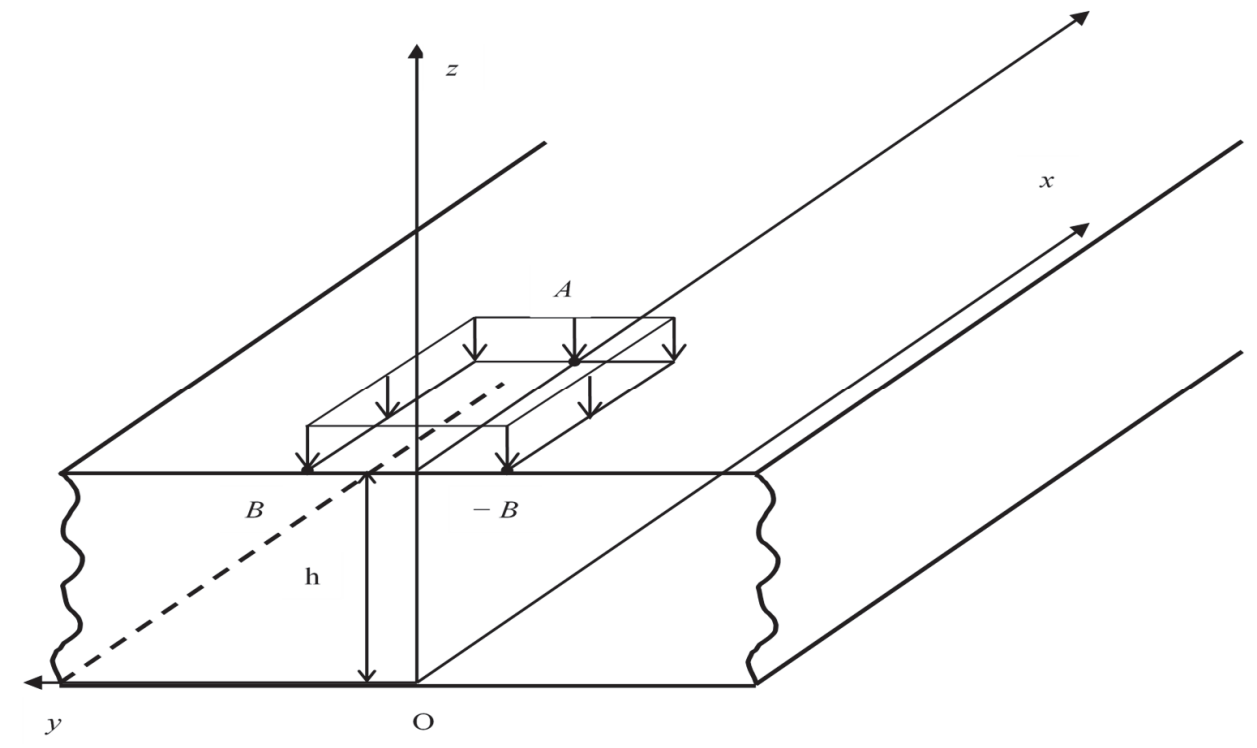

Figure 1: Geometric model of the problem

The conditions of an ideal contact are given at the layer's edge $x=0$ and at the lower face $z=0-$ by this, the authors mean frictionless contact conditions. At the same time, these faces are thermo-isolated. The known temperature $T(x, y, z)$, found earlier from the corresponding thermoconductivity problem, and pressure load $p(x, y)$ both 
distributed on the segments $x \in[0, A], y \in[-B, B]$ are applied at the upper face $z=b$. The proper weight of the layer should be taken into consideration.

The stated problem for the semi-infinite layer is formulated in terms of the boundary value problem

$$
\begin{aligned}
& G \Delta \mathrm{u}^{*}+(\lambda+G) \Theta^{\prime}+X(x, y, z)=(3 \lambda+2 G) \alpha T^{\prime} \\
& G \Delta \mathrm{v}^{*}+(\lambda+G) \Theta^{\prime}+Y(x, y, z)=(3 \lambda+2 G) \alpha T^{\prime} \\
& G \Delta \mathrm{w}^{*}+(\lambda+G) \Theta^{\prime}+Z(x, y, z)=(3 \lambda+2 G) \alpha T^{\prime}
\end{aligned}
$$

Here $\Delta=\frac{\partial^{2}}{\partial x^{2}}+\frac{\partial^{2}}{\partial y^{2}}+\frac{\partial^{2}}{\partial z^{2}}$ is a Laplace's differential operator, $\mathrm{u}^{*}=u_{x}(x, y, z), \mathrm{v}^{*}=u_{y}(x, y, z), \mathrm{w}^{*}=u_{z}(x, y, z)-$ unknown displacements, $\frac{\partial f}{\partial x}=f^{\prime}, \frac{\partial f}{\partial y}=f^{\prime}, \frac{\partial f}{\partial z}=f^{\prime}, X, Y, Z$ are the components of the proper weight force. $\lambda, G$ are Lame's constants and $\Theta=\mathrm{u}^{\prime}(x, y, z)+\mathrm{v}^{\prime}(x, y, z)+\mathrm{w}^{\prime}(x, y, z)$ is a volume expansion, $\alpha$ is a coefficient of linear expansion.

One must solve the boundary value problem with the following boundary conditions. The conditions of an ideal contact are given at the layer's edge $x=0$ and at the lower face $z=0$

$$
\begin{aligned}
& \left.\mathrm{u}^{*}\right|_{x=0}=0,\left.\tau_{x z}\right|_{x=0}=0,\left.\tau_{x y}\right|_{x=0}=0 \\
& \left.\mathrm{w}^{*}\right|_{z=0}=0,\left.\tau_{z x}\right|_{z=0}=0,\left.\tau_{z y}\right|_{z=0}=0
\end{aligned}
$$

The pressure load $p(x, y)$ distributed on the segments $x \in[0, A], y \in[-B, B]$ is applied at the upper face $z=b$

$$
\left.\sigma_{z}\right|_{z=h}=-p(x, y),\left.\tau_{z x}\right|_{z=h}=0,\left.\tau_{z y}\right|_{z=h}=0
$$

The authors propose to solve the previously the stated problem for the subcase when the unit normal loading is applied to an arbitrary point on the upper face of the layer. The solution to this problem could be used as an influence function to construct the solution for the stated loading. For this subcase the boundary conditions (5) take the form

$$
\left.\sigma_{z}\right|_{z=b}=-\delta(x-a) \delta(y-b),\left.\tau_{z x}\right|_{z=b}=0,\left.\tau_{z y}\right|_{z=h}=0
$$

$\delta(x)$ - Dirac's function.

The field of displacements and stress should be found. The temperature $T(x, y, z)$, on the right hand parts of Lame's equations (2), is unknown. It's needed to solve the corresponding thermoconductivity problem for the layer (1) with the same Lame's constants. These values will be used as known in further calculations.

\section{STATEMENT AND SOLVING THE CORRESPONDING THERMOCONDUCTIVITY PROBLEM FOR A SEMI- INFINITE LAYER}

I t's necessary to construct a solution to Laplace's equation decreasing on infinity

$$
\Delta T(x, y, z)=0, \Delta=\frac{\partial^{2}}{\partial x^{2}}+\frac{\partial^{2}}{\partial y^{2}}+\frac{\partial^{2}}{\partial z^{2}}
$$

It is supposed that edges $x=0$ and $z=0$ are thermo isolated 


$$
\left.\frac{\partial T(x, y, z)}{\partial x}\right|_{x=0}=0,\left.\frac{\partial T(x, y, z)}{\partial z}\right|_{z=0}=0
$$

Here function $T(x, y, z)$ is the unknown temperature of the layer.

At the upper face $z=b$ of the layer, the temperature is given along the segment $x \in[0, A], y \in[-B, B]$

$$
T(x, y, h)=f(x, y), x \in[0, A], y \in[-B, B]
$$

The integral transform method is used to derive the one-dimensional boundary problem. The Fourier cosine transform with regard to the variable $x$ and full Fourier's transform with regard to the variable $y$ are applied consecutively to the Laplace equation $(7)$ and boundary conditions $(8,9)$. It leads to the one-dimensional problem at the transforms' domain

$$
\begin{aligned}
& T_{\alpha \beta}^{\prime \prime}(z)-N^{2} T_{\alpha \beta}(z)=0, z \in(0, h) \\
& T_{\alpha \beta}^{\prime}(0)=0, T_{\alpha \beta}(b)=f_{\alpha \beta}
\end{aligned}
$$

where $f_{\alpha \beta}=\int_{-\infty}^{\infty} \int_{0}^{\infty} f(x, y) \cos \alpha x \cdot e^{i \beta y} d y d x, N^{2}=\alpha^{2}+\beta^{2}, \alpha, \beta$ are Fourier's transform parameters. Usage of the general solution of the equation (10)

$$
T_{\alpha \beta}(₹)=C_{1} s h N_{z}+C_{2} c b N z, C_{1}=0, C_{2}=f_{\alpha \beta} / c b N h
$$

leads to the final solution in the transform domain, where the inverse integral transform was applied

$$
T(x, y, z)=\frac{2}{\pi} \frac{1}{2 \pi} \int_{0}^{\infty} \int_{-\infty}^{\infty} f_{\alpha \beta} \cdot \frac{c h N z}{c h N b} \cdot \cos \alpha x \cdot e^{-i \beta y} d \alpha d \beta
$$

The final solution of the stated conductivity problem (7-9) is presented in the form

$$
T(x, y, z)=\frac{2 C}{\pi N} \sum_{k=1}^{N} \int_{0}^{\infty} \frac{c b(t z)}{\operatorname{ch}(b t)} \frac{\sin \left(t A \sqrt{1-\tau_{k}^{2}}\right) \sin \left(t B \tau_{k}\right)}{t A \sqrt{1-\tau_{k}^{2}} B \tau_{k}} \cos \left(t x \sqrt{1-\tau_{k}^{2}}\right) \cos \left(t y \tau_{k}\right) d t
$$

where $\tau_{k}^{(N)}$ are zeros of Chebyshov polynomials of the first kind, $C$ is the constant value of temperature, distributed along the segment $x \in[0, A], y \in[-B, B]$.

A more detailed derivation of the formula (12) is given in the Appendix 1.

An important property

$$
\frac{C}{2 \pi N} \sum_{k=1}^{N} \frac{1}{\tau_{k} \sqrt{1-\tau_{k}^{2}}} \ln \left(\frac{\sqrt{1-\tau_{k}^{2}}+\frac{B}{A} \tau_{k}}{\sqrt{1-\tau_{k}^{2}}-\frac{B}{A} \tau_{k}}\right)^{2}=C
$$

was obtained based on solution (12) and will be used for calculating stress at the corner point of a layer. A more detailed derivation of the formula (13) is given in Appendix 2.

The next step is to find the solution of the problem for the elastic layer $-\infty<x<\infty,-\infty<y<\infty, 0<z<b$ under its proper weight. 


\section{THE DERIVING OF NORMAL STRESS WITH REGARD TO ITS PROPER WEIGHT}

o solve this problem, the displacements $\mathrm{u}^{\gamma}, \mathrm{v}^{\gamma}, \mathrm{w}^{\gamma}$ will be searched as the function depending on the variable $z$
$\mathrm{u}^{\gamma}=f_{0}(z), \mathrm{v}^{\gamma}=f_{1}(z), \mathrm{w}^{\gamma}=f_{2}(z)$

Because of problem's symmetry one can take $\mathrm{u}^{\gamma} \equiv 0, \mathrm{v}^{\gamma} \equiv 0$. Hence, the tangent stress $\tau_{x y}=0$. That's why the displacements were taken in the form (14), the boundary condition of the ideal contact (3) is satisfied automatically at the edge of the layer.

The function $\mathrm{w}^{\gamma}$ satisfies the Lame's equation

$$
\Delta \mathrm{w}^{\gamma}+\mu_{0}\left(\Theta+\mathrm{w}^{\gamma}\right)^{\prime}+\gamma=0
$$

where $\gamma=q_{\tau} / G, q_{z}$ is the weight of an elastic material, $\mu_{0}=(1-2 \mu)^{-1}$.

The face $z=b$ is supposed free of stress

$$
\left.\sigma_{z=b}^{\gamma}\right|_{z=h}=0,\left.\tau_{æ x}\right|_{z=h}=0,\left.\tau_{\S y}\right|_{z=h}=0
$$

The lower face $z=0$ is in ideal contact conditions

$$
\left.\mathrm{w}^{\gamma}\right|_{z=0}=0,\left.\tau_{z x}\right|_{z=0}=0,\left.\tau_{\mathfrak{y y}}\right|_{z=0}=0
$$

Finally, one gets the boundary problem

$$
\begin{aligned}
& \left(1+\mu_{0}\right) f_{2}^{\prime \prime}(z)+\gamma=0,0<z<b \\
& f_{2}(0)=0, f_{2}^{\prime}(b)=0
\end{aligned}
$$

The solution has the form

$$
\mathrm{w}^{\gamma}=\left(1+\mu_{0}\right)^{-1} \gamma\left(b z-z^{2} / 2\right)
$$

Hence, the stress $\sigma_{x}^{\gamma}$ for the layer with regard to its proper weight is constructed

$$
\sigma_{x}^{\gamma}=\mu(1-\mu)^{-1} q_{z}(b-z)
$$

The solution of the initial stated problem (2-5) will be searched in the form

$$
\mathrm{u}^{*}=\mathrm{u}+\mathrm{u}^{\gamma}, \mathrm{v}^{*}=\mathrm{v}+\mathrm{v}^{\gamma}, \mathrm{w}^{*}=\mathrm{w}+\mathrm{w}^{\gamma}, \sigma_{x}^{*}=\sigma_{x}+\sigma_{x}^{\gamma}
$$

Here $\mathrm{u}(x, y, z), \mathrm{v}(x, y, z), \mathrm{w}(x, y, z), \sigma_{x}(x, y, z)$ are displacements and stress appearing in a body without regard to its proper weight. 


\section{REDUCTION OF THE INITIALLY STATED UNCOUPLED THERMOELASTICITY PROBLEM TO A ONE- DIMENSIONAL VECTOR BOUNDARY PROBLEM}

$\mathrm{O}$ ne neglects the volume forces $X, Y, Z$. So, Lame's equations (2) can be written in the vector form

$$
\Delta(\mathrm{u}, \mathrm{v}, \mathrm{w})+\mu_{0}\left(\Theta^{\prime}, \Theta^{*}, \Theta^{\prime}\right)=\rho\left(T^{\prime}, T^{*}, T^{\prime}\right)
$$

here $\rho=2 \alpha \mu_{0}(1+\mu)=\alpha \mu_{1}^{-1} \mu_{0}=\alpha \mu_{*}$.

The boundary conditions are

$$
\begin{gathered}
\left.\sigma_{z}\right|_{z=h}=-\delta(x-a) \delta(y-b),\left.\tau_{z x}\right|_{z=h}=0,\left.\tau_{z y}\right|_{z=b}=0 \\
\left.\mathrm{u}\right|_{x=0}=0,\left.\tau_{x \chi}\right|_{x=0}=0,\left.\tau_{x y}\right|_{x=0}=0 \\
\left.\mathrm{w}\right|_{z=0}=0,\left.\tau_{x x}\right|_{z=0}=0,\left.\tau_{y y}\right|_{z=0}=0
\end{gathered}
$$

Let's reformulate the boundary conditions (19) in terms of displacements, taking into consideration well known formulas connecting the displacements and stresses [20]

$$
\begin{aligned}
& \mu\left[\mathrm{u}^{\prime}(x, y, h)+\mathrm{v}^{\cdot}(x, y, h)\right]+(1-\mu) \mathrm{w}^{\prime}(x, y, h)-\rho\left(2 \mu_{0}\right)^{-1} T(x, y, h)=-\delta(x-a) \delta(y-b) /\left(2 G \mu_{0}\right) \\
& \mathrm{u}^{\prime}(x, y, h)+\mathrm{w}^{\prime}(x, y, h)=0, \mathrm{w}^{\cdot}(x, y, h)+\mathrm{v}^{\prime}(x, y, h)=0 \\
& \mathrm{u}(0, y, z)=\mathrm{v}^{\prime}(0, y, z)=\mathrm{w}^{\prime}(0, y, z)=0 \\
& \mathrm{w}(x, y, 0)=0, \mathrm{u}^{\prime}(x, y, 0)+\mathrm{w}^{\prime}(x, y, 0)=0, \mathrm{w}^{\cdot}(x, y, 0)+\mathrm{v}^{\prime}(x, y, 0)=0 .
\end{aligned}
$$

The idea is to reduce the Lame's equations (18) to one independently and two simultaneously solving equations, subjected by separated conditions (20). The unknown functions

$$
Z(x, y, z)=\mathrm{u}^{\prime}(x, y, z)+\mathrm{v}^{\cdot}(x, y, z), \mathrm{S}(x, y, z)=\mathrm{v}^{\prime}(x, y, z)-\mathrm{u}^{\cdot}(x, y, z)
$$

are input accordingly to [11]. The Lame's equations system (18) is separated on the system of two equations

$$
\begin{aligned}
\Delta Z+\mu_{0} \nabla_{x y}\left(Z+\mathrm{w}^{\prime}\right) & =\rho \nabla_{x y} T, \nabla_{x y}=\frac{\partial^{2}}{\partial x^{2}}+\frac{\partial^{2}}{\partial y^{2}} \\
\Delta \mathrm{w}+\mu_{0}\left(Z+\mathrm{w}^{\prime}\right)^{\prime} & =\rho T
\end{aligned}
$$

and independently solving equation $\Delta S=0$.

The boundary conditions (20) after separation procedure will take the form with regard to the new functions (21)

$$
\begin{aligned}
& Z^{\prime}(0, y, z)=0, \mathrm{w}^{\prime}(0, y, z)=0, S(0, y, z)=0 \\
& Z^{\prime}(x, y, 0)=0, \mathrm{w}(x, y, 0)=0, S(x, y, 0)=0 \\
& \nabla_{x y} \mathrm{w}(x, y, b)+Z^{\prime}(x, y, h)=0, S^{\prime}(x, y, b)=0
\end{aligned}
$$




$$
\mu Z(x, y, h)+(1-\mu) \mathrm{w}^{\prime}(x, y, h)=-\left(2 G \mu_{0}\right)^{-1} \delta(x-a) \delta(y-b)+\rho\left(2 \mu_{0}\right)^{-1} T(x, y, h) .
$$

In the domain of Fourier's transforms (the cosine transform with regard to the variable $x$ and full Fourier transform with regard to the variable $y$ ) one can derive two boundary problems. First, it is formulated for the unknown function $S(x, y, z)$

$$
S_{\beta \alpha}^{\prime \prime}(z)-N^{2} S_{\beta \alpha}(z)=0, \quad 0<z<h, \quad S_{\beta \alpha}{ }^{\prime}(b)=0, \quad S_{\beta \alpha}(0)=0
$$

It's obvious, that this problem has a trivial solution $S_{\beta \alpha}(z)=0$, and, hence, $S(x, y, z)=0$.

For the functions $Z_{\beta \alpha}(z), \mathrm{w}_{\beta \alpha}(₹)$ the one dimensional boundary problem is stated

$$
\begin{gathered}
L_{2} \overrightarrow{\mathbf{y}}(z)=\mathbf{f}(z), 0<z<h \\
U_{0}[\overrightarrow{\mathbf{y}}(z)]=\boldsymbol{\gamma}_{0}, U_{1}[\overrightarrow{\mathbf{y}}(z)]=\boldsymbol{\gamma}_{1}
\end{gathered}
$$

here $L_{2}$ is the differential operator of second order

$$
L_{2} \overrightarrow{\mathbf{y}}(z) \equiv \mathbf{I} \overrightarrow{\mathbf{y}}^{\prime \prime}(z)+\mu_{0} \mathbf{Q} \overrightarrow{\mathbf{y}}^{\prime}(z)-N^{2} \mathbf{P} \overrightarrow{\mathbf{y}}(z)=\mathbf{f}(z), 0<z<b
$$

$\mathbf{I}$ is unit matrix, $\mathbf{Q}=\left(\begin{array}{cc}0 & \mu_{*}^{-1} \\ -N_{\alpha \beta}^{2} & 0\end{array}\right), \mathbf{P}=\left(\begin{array}{cc}\mu_{*}^{-1} & 0 \\ 0 & \mu_{*}\end{array}\right), \mathbf{f}(z)=\rho\left(\begin{array}{c}T_{\beta \alpha}^{\prime}(z) \\ -N^{2} T_{\beta \alpha}(z)\end{array}\right), \overrightarrow{\mathbf{y}}(z)=\left(\begin{array}{c}w_{\beta \alpha}(z) \\ z_{\beta \alpha}(z)\end{array}\right)$ is the unknown vector of transforms.

The boundary functionals $U_{i}, i=0,1$ are written in the corresponding forms

$$
\begin{aligned}
& U_{0}[\mathbf{y}]=\mathbf{I}_{1} \overrightarrow{\mathbf{y}}(z)+\mathbf{I}_{2} \overrightarrow{\mathbf{y}^{\prime}}(z)=\boldsymbol{\gamma}_{0} \\
& U_{1}[\mathbf{y}]=\mathbf{A} \overrightarrow{\mathbf{y}}(z)+\overrightarrow{\mathbf{B y}^{\prime}}(z)=\boldsymbol{\gamma}_{1} \\
& \mathbf{I}_{1}=\left(\begin{array}{ll}
1 & 0 \\
0 & 0
\end{array}\right), \mathbf{I}_{2}=\left(\begin{array}{ll}
0 & 0 \\
0 & 1
\end{array}\right), \mathbf{A}=\left(\begin{array}{cc}
-N^{2} & 0 \\
0 & \mu
\end{array}\right), \mathbf{B}=\left(\begin{array}{cc}
0 & 1 \\
1-\mu & 0
\end{array}\right) \\
& \boldsymbol{\gamma}_{0}=0, \boldsymbol{\gamma}_{1}=\left(\begin{array}{c}
0 \\
-\left(2 G \mu_{0}\right)^{-1} \cos \alpha a \cdot e^{i \beta b}+\rho\left(2 \mu_{0}\right)^{-1} T_{\beta \alpha}(b)
\end{array}\right) .
\end{aligned}
$$

The solution of the problem (24) is searched in the form [37]

$$
\overrightarrow{\mathbf{y}}(z)=\left(\begin{array}{c}
\mathrm{w}_{\beta \alpha}(z) \\
Z_{\beta \alpha}(z)
\end{array}\right)=\int_{0}^{b} \mathbf{G}(z, \xi) \mathbf{f}(\xi) d \xi+\Psi_{0}(z) \boldsymbol{\gamma}_{0}+\Psi_{1}(z) \boldsymbol{\gamma}_{1}
$$

where $\mathbf{G}(z, \xi)$ is Green matrix function, $\Psi_{i}(₹), i=0,1$ are the basis matrices. 


\section{THE CONSTRUCTION OF THE MATRIX FUNDAMENTAL SOLUTION SYSTEM, THE MATRIX BASIC SOLUTION SYSTEM, THE FUNDAMENTAL MATRIX AND GREEN MATRIX FUNCTION}

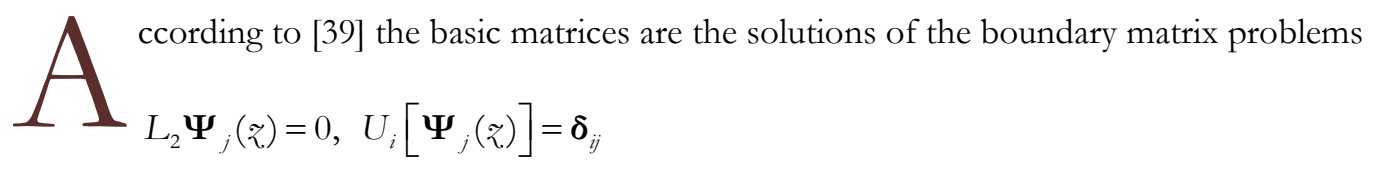

$\boldsymbol{\delta}_{i j}$ is Kronecker's symbol.

The basic matrices are constructed in the form

$$
\Psi_{i}(z)=\mathbf{Y}_{-}(z) \mathbf{C}_{i}^{0}+\mathbf{Y}_{+}(z) \mathbf{C}_{i}^{1}, \quad i=0,1
$$

where $\mathbf{Y}_{-}(z), \mathbf{Y}_{+}(z)$ are the matrix fundamental solutions of the homogeneous equation (26) correspondingly decreasing and increasing in infinity.

The algorithm of fundamental solutions $\mathbf{Y}_{\mp}(z)$ derivation is described in [37], where the method of fundamental matrix equation is also indicated. According to this approach, the solution is searched as the contour integral $[39,40]$

$$
\mathbf{Y}(z)=\frac{1}{2 \pi i} \int_{C} e^{s z} \mathbf{M}^{-1}(s) d s
$$

where contour $C$ covers all poles of the matrix $\mathbf{M}^{-1}(s)$, where $\mathbf{M}(s)=\mathbf{I} s^{2}+\mu_{0} \mathbf{Q} s-N^{2} \mathbf{P}, \operatorname{det} \mathbf{M}(s)=\left(s^{2}-N^{2}\right)^{2}$, $s= \pm N$. Let's calculate matrix $\mathbf{M}^{-1}(s)=\mathbf{M}^{*}(s) / \operatorname{det} \mathbf{M}(s)$, so (27) will be transformed

$$
\mathbf{Y}(z)=\frac{1}{2 \pi i} \int_{C} \frac{e^{s z}}{\left(s^{2}-N^{2}\right)^{2}} \mathbf{M}^{*}(s) d s
$$

here $\mathbf{M}^{*}(s)$ is a union matrix. With the help of the residual theorem, one derives

$$
\begin{gathered}
\mathbf{Y}(z)=\sum_{j=0}^{2} \boldsymbol{\Gamma}^{(j)}\left(\frac{d}{d z}\right)^{j} y_{\mp}(z), \quad y_{\mp}(z)=\operatorname{Re} s \frac{e^{s z}}{(s-N)^{2}(s+N)^{2}}, s=\mp N \\
y_{-}(z)=\left(4 N^{3}\right)^{-1} e^{-N z}\left(N_{z}+1\right), y_{+}(z)=\left(4 N^{3}\right)^{-1} e^{N z}(N z-1)
\end{gathered}
$$

Here, to find matrices $\boldsymbol{\Gamma}^{(j)}, j=\overline{0,2}$ one uses the fact, that $\mathbf{M}^{-1}(s)=\frac{1}{p_{4}(s)} \sum_{i=0}^{2} \boldsymbol{\Gamma}^{(i)} s^{i}, p_{4}(s)=s^{4}-2 s^{2} N^{2}+N^{4}$ or $\mathbf{I} \cdot p_{4}(s)=\mathbf{M}(s) \sum_{i=0}^{2} \boldsymbol{\Gamma}^{(i)} s^{i}$. Using that coefficient near the same exponents should be equal, the matrixes $\boldsymbol{\Gamma}^{(j)}, j=\overline{0,2}$ are found

$$
\boldsymbol{\Gamma}^{(2)}=\mathbf{I}, \boldsymbol{\Gamma}^{(1)}=-\mu_{0} \mathbf{Q}, \boldsymbol{\Gamma}^{(0)}=-N^{2} \mathbf{P}^{-1} .
$$

As a result, the fundamental matrix system is derived

$$
\mathbf{Y}_{\mp}(\mathrm{z})=\mu_{0} e^{\mp N_{z}}\left(\begin{array}{cc}
-N_{\varkappa} \mp \kappa & \pm \mu_{*}^{-1} ₹ \\
\mp N^{2} ₹ & \mu_{*}^{-1}\left(N_{\varkappa} \mp \kappa\right)
\end{array}\right)
$$




$$
\kappa=3-4 \mu .
$$

The unknown constant matrices $\mathbf{C}_{i}^{0}, \mathbf{C}_{i}^{1}, i=0,1$ and $\mathbf{Y}_{\mp}^{\prime}(z)$ are shown in Appendix 3.

The basic matrices are expressed by the formulas after transformations

$$
\Psi_{i}(z)=\frac{1}{D_{N}}\left(\begin{array}{cc}
\psi_{i}^{(11)} & \psi_{i}^{(12)} \\
\psi_{i}^{(21)} & \psi_{i}^{(22)}
\end{array}\right)
$$

where $D_{N}=2 s h(2 N h)+4 N h$. The components of the matrix (28) are shown in Appendix 4.

The expression for Green's matrix function is proposed in [39]

$$
\mathbf{G}(z, \xi)=\boldsymbol{\Phi}(z, \xi)-\Psi_{0}(z) \cdot U_{0}[\boldsymbol{\Phi}(z, \xi)]-\Psi_{1}(z) \cdot U_{1}[\mathbf{\Phi}(z, \xi)]
$$

where $\boldsymbol{\Phi}(z, \xi)$ is a fundamental matrix of the corresponding homogeneous equation (26).

For the construction of the fundamental matrix $\mathbf{\Phi}(z, \xi)$, let's continue the function $y(z)$ on all the real axes and apply the Fourier integral transform

$$
y_{\sigma}=\int_{-\infty}^{\infty} y(z) e^{i \sigma z} d z
$$

The same transform (30) should be applied to the equation in (24)

$$
-\sigma^{2} \mathbf{I} y_{\sigma}-\mu_{0} \mathbf{Q} i \sigma y_{\sigma}-N^{2} \mathbf{P} y_{\sigma}=\mathbf{f}_{\sigma}
$$

Hence, one derives

$$
y_{\sigma}=\mathbf{M}^{-1}(-i \sigma) \mathbf{f}_{\sigma}
$$

After the inverse integral Fourier transform the correspondence for $y(z)$ is obtained

$$
y(z)=\frac{1}{2 \pi} \int_{-\infty}^{\infty} \int_{-\infty}^{\infty} \mathbf{M}^{-1}(-i \sigma) \mathbf{f}_{\sigma} e^{-i \sigma(z-\xi)} d \sigma d \xi
$$

According to the fundamental matrix definition, this matrix is

$$
\boldsymbol{\Phi}(z-\xi)=\frac{1}{2 \pi} \int_{-\infty}^{\infty} \mathbf{M}^{-1}(-i \sigma) e^{-i \sigma(z-\xi)} d \sigma
$$

Or after changing the variable $s=-i \sigma$, the matrix has the form

$$
\boldsymbol{\Phi}(z-\xi)= \pm \frac{1}{2 \pi i} \int_{C_{ \pm}} \frac{\mathbf{M}^{*}(s)}{\left(s^{2}-N^{2}\right)^{2}} e^{s(z-\xi)}, \begin{aligned}
& z-\xi>0 \\
& z-\xi<0
\end{aligned}
$$

here $C_{ \pm}$are the closed contours that cover the poles $s= \pm|N|$ or $\sigma=\mp i N$. The residuals at $\sigma=i N$ and $\sigma=-i N$ are correspondingly equal

$$
\varphi_{+}=e^{N y}\left(4 N^{3}\right)^{-1}(1-N y), \varphi_{-}=e^{-N y}\left(4 N^{3}\right)^{-1}(1+N y), y=z-\xi
$$


The matrices $\boldsymbol{\Phi}_{ \pm}$are searched with the help of the matrices (32)

$$
\boldsymbol{\Phi}_{ \pm}=\mathbf{I} \varphi_{ \pm}^{\prime \prime}-\mu_{0} \mathbf{Q} \varphi_{ \pm}^{\prime}-N^{2} \mathbf{P} \varphi_{ \pm}
$$

After substitution of the expressions (33) into the equations (34), uniting these results, the fundamental matrix is constructed in the form

$$
\boldsymbol{\Phi}(z, \xi)=\frac{\mu_{0} e^{-N|z-\xi|}}{4 N}\left(\begin{array}{cc}
-N|z-\xi|-\kappa & \mu_{*}^{-1}(z-\xi) \\
-N^{2}(z-\xi) & \mu_{*}^{-1}(N|z-\xi|-\kappa)
\end{array}\right)
$$

According to the formula (29) the Green's matrix has the form

$$
\mathbf{G}(z, \xi)=\frac{\mu_{0} e^{-N \mid z-\xi}}{4 N}\left(\begin{array}{cc}
-(N|z-\xi|+\kappa) & \mu_{*}^{-1}(z-\xi) \\
-N^{2}(z-\xi) & \mu_{*}^{-1}\left(\left.N\right|_{z}-\xi \mid-\kappa\right)
\end{array}\right)-\frac{\mu_{0}}{2 N D_{N}}\left(\begin{array}{cc}
\phi^{(11)} & \phi^{(12)} \\
\phi^{(21)} & \phi^{(22)}
\end{array}\right)
$$

where components $\phi^{(i j)}, i, j=1,2$ are shown at Appendix 4.

Finally, the solution of the vector boundary problem (24) in Fourier's transform domain is constructed with the formulas

$$
\begin{aligned}
\mathrm{w}_{\beta \alpha}(z)= & -\frac{\mu_{0} \rho}{4 N} \int_{0}^{b} e^{-N|z-\xi|}\left\{(N|z-\xi|+\kappa) T_{\beta \alpha}^{\prime}(\xi)+\mu_{*}^{-1}(z-\xi) N^{2} T_{\beta \alpha}(\xi)\right\} d \xi- \\
& -\frac{\mu_{0} \rho}{2 N D_{N}} \int_{0}^{b}\left\{\phi^{(11)} T_{\beta \alpha}^{\prime}(\xi)-N^{2} \phi^{(12)} T_{\beta \alpha}(\xi)\right\} d \xi+\frac{\cos \alpha a \cdot e^{i \beta b}}{2 G D_{N}} F_{1}(N, z)-\frac{\rho \cdot T_{\beta \alpha}(b)}{2 D_{N}} F_{1}(N, z) \\
Z_{\beta \alpha}(z)= & -\frac{\mu_{0} \rho}{4} \int_{0}^{b} e^{-N|z-\xi|} N\left\{(z-\xi) T_{\beta \alpha}^{\prime}(\xi)+\mu_{*}^{-1}(N|z-\xi|-\kappa) T_{\beta \alpha}(\xi)\right\} d \xi- \\
& -\frac{\mu_{0} \rho}{2 N D_{N}} \int_{0}^{b}\left\{\phi^{(21)} T_{\beta \alpha}^{\prime}(\xi)-N^{2} \phi^{(22)} T_{\beta \alpha}(\xi)\right\} d \xi+\frac{\cos \alpha a \cdot e^{i \beta b}}{2 G D_{N}} F_{2}(N, z)-\frac{\rho \cdot T_{\beta \alpha}(b)}{2 D_{N}} F_{2}(N, z)
\end{aligned}
$$

where

$$
\begin{aligned}
& \frac{1}{2} F_{1}(N, z)=(z+b) \operatorname{sh} N(b-z)-(b-z) \operatorname{sh} N(b+z)-\mu_{1}^{-1} \frac{1}{N}(\operatorname{cb} N(b+z)-\operatorname{sh} N(b-z)) \\
& \frac{1}{2} F_{2}(N, z)=N(z+b) \operatorname{cb} N(b-z)+N(b-z) \operatorname{cb} N(b+z)-\mu_{0}^{-1}(\operatorname{sb} N(b+z)+\operatorname{sb} N(b-z)) \\
& D_{N}=2 \operatorname{sh}(2 N h)+4 N b
\end{aligned}
$$

\section{DERIVING THE DISPLACEMENTS AND STRESS FORMULAS IN THE TRANSFORM DOMAIN AND THEIR INVERSION}

$\mathrm{S}$

$\mathrm{o}$, the transformation of the solution is constructed in the form of superposition

$$
\mathrm{w}_{\beta \alpha}(z)=\sum_{k=1}^{3} \mathrm{w}_{\beta \alpha}^{k}(z), \quad Z_{\beta \alpha}(z)=\sum_{k=1}^{3} Z_{\beta \alpha}^{k}(z)
$$

where 


$$
\begin{aligned}
& \left(\begin{array}{l}
\mathrm{w}_{\beta \alpha}^{1}(z) \\
Z_{\beta \alpha}^{1}(z)
\end{array}\right)=\frac{\cos \alpha a \cdot e^{i \beta b}}{2 G D_{N}}\left(\begin{array}{l}
F_{1}(N, z) \\
F_{2}(N, z)
\end{array}\right), \\
& \left(\begin{array}{c}
\mathrm{w}_{\beta \alpha}^{2}(z) \\
Z_{\beta \alpha}^{2}(z)
\end{array}\right)=-\frac{\rho \cdot T_{\beta \alpha}(b)}{2 D_{N}}\left(\begin{array}{l}
F_{1}(N, z) \\
F_{2}(N, z)
\end{array}\right),
\end{aligned}
$$

functions $F_{1}(N, z), F_{2}(N, z), D_{N}$ are defined in (37).

$$
\begin{aligned}
\mathrm{w}_{\beta \alpha}^{3}(z)= & -\frac{\mu_{0} \rho}{4 N} \int_{0}^{b} e^{-N \mid z-\xi}\left\{(N|z-\xi|+\kappa) T_{\beta \alpha}^{\prime}(\xi)+\mu_{*}^{-1}(z-\xi) N^{2} T_{\beta \alpha}(\xi)\right\} d \xi- \\
& -\frac{\mu_{0} \rho}{2 N D_{N}} \int_{0}^{b}\left\{\phi^{(11)} T_{\beta \alpha}^{\prime}(\xi)-N^{2} \phi^{(12)} T_{\beta \alpha}(\xi)\right\} d \xi, \\
Z_{\beta \alpha}^{3}(z)= & -\frac{\mu_{0} \rho}{4} \int_{0}^{b} e^{-N|z-\xi|} N\left\{(z-\xi) T_{\beta \alpha}^{\prime}(\xi)+\mu_{*}^{-1}(N|z-\xi|-\kappa) T_{\beta \alpha}(\xi)\right\} d \xi- \\
& -\frac{\mu_{0} \rho}{2 N D_{N}} \int_{0}^{b}\left\{\phi^{(21)} T_{\beta \alpha}^{\prime}(\xi)-N^{2} \phi^{(22)} T_{\beta \alpha}(\xi)\right\} d \xi .
\end{aligned}
$$

In detail, the inversion of the Fourier transform for terms of the form (39) is described using the example of the term $\mathrm{w}_{\beta \alpha}^{2}(z)$. Proceeding from the boundary condition in (10) of the stationary heat conduction problem considered in part 3 of the article, one gets $T_{\beta \alpha}(b)=f_{\beta \alpha}=\int_{-\infty}^{\infty} \int_{0}^{\infty} f(\xi, \eta) e^{i \beta \eta} \cos \alpha \xi d \eta d \xi$. Then the representation of the term $\mathrm{w}_{\beta \alpha}^{2}(z)$ will take the form

$$
\mathrm{w}^{2}(x, y, z)=-\frac{\rho}{2 \pi^{2}} \int_{-\infty}^{\infty} \int_{0}^{\infty} \frac{F_{1}(N, z)}{D_{N}} \int_{-\infty}^{\infty} \int_{0}^{\infty} f(\xi, \eta) e^{i \beta \eta} \cos \alpha \xi \cdot e^{-i \beta y} \cos \alpha x d \beta d \alpha d \eta d \xi
$$

Changing the order of integration, using the formula (401.06.,[41]), and application of the Euler formula allows to simplify the term $\mathrm{w}_{\beta \alpha}^{2}(z)$. The temperature is assumed to be constant, distributed over a finite interval $-B<\eta<B, 0<\xi<A$, which leads to the expression

$$
\mathrm{w}^{2}(x, y, z)=-\frac{\rho \cdot C}{8 \pi^{2}} \int_{-B}^{B} \int_{0}^{A} \int_{-\infty}^{\infty} \int_{-\infty}^{\infty} \frac{F_{1}(N, z)}{D_{N}} e^{-i \beta(y-\eta)}\left(e^{-i \alpha(x+\xi)}+e^{-i \alpha(x-\xi)}\right) d \eta d \xi d \beta d \alpha
$$

Further, using formula (A3) in the Appendix 1, correspondence (41) can be written as

$$
\mathrm{w}^{2}(x, y, z)=-\frac{\rho \cdot C}{4 \pi} \int_{-B}^{B} \int_{0}^{A} \int_{0}^{\infty} \frac{t \cdot F_{1}(t, z)}{D_{t}} \cdot J_{0}^{*}(t, x, y, \xi, \eta) d \eta d \xi d t, D_{t}=s h(2 t)+2 t
$$

Applying the procedure for calculating multiple integrals of the Bessel function to internal integral, one gets

$$
\mathrm{w}^{2}(x, y, z)=-\frac{2 \rho \cdot C}{\pi^{2}} \int_{0}^{\frac{\pi}{2}} \int_{0}^{\infty} \frac{t \cdot F_{1}(t, z)}{D_{t}} S_{t}^{A, B}(\psi) \cos (t x \cos \psi) \cos (t y \sin \psi) d \psi d t
$$


The formulas

$$
\mathrm{u}_{\beta \alpha}(z)=\alpha N^{-2} Z_{\beta \alpha}(z), \mathrm{v}_{\beta \alpha}(z)=i \beta N^{-2} Z_{\beta \alpha}(z)
$$

were used to find the originals of the displacements $u^{2}(x, y, z), v^{2}(x, y, z)$

So,

$$
\mathrm{u}_{\beta \alpha}^{2}(z)=-\frac{\rho \cdot T_{\beta \alpha}(b)}{2 D_{N}} \frac{\alpha}{N^{2}} F_{2}(N, z), \quad v_{\beta \alpha}^{2}(z)=-\frac{\rho \cdot T_{\beta \alpha}(b)}{2 D_{N}} \frac{i \beta}{N^{2}} F_{2}(N, z)
$$

By analogy, it was found

$$
\begin{aligned}
& \mathrm{u}^{2}(x, y, z)=\frac{2 \rho \cdot C}{\pi^{2}}\left(\frac{\partial}{\partial x}\right) \int_{0}^{\frac{\pi}{2}} \int_{0}^{\infty} \frac{F_{2}(t, z)}{t \cdot D_{t}} S_{t}^{A, B}(\psi) \cos (t x \cos \psi) \cos (t y \sin \psi) d \psi d t, \\
& \mathrm{v}^{2}(x, y, z)=\frac{2 \rho \cdot C}{\pi^{2}}\left(\frac{\partial}{\partial y}\right) \int_{0}^{\frac{\pi}{2}} \int_{0}^{\infty} \frac{F_{2}(t, z)}{t \cdot D_{t}} S_{t}^{A, B}(\psi) \cos (t x \cos \psi) \cos (t y \sin \psi) d \psi d t .
\end{aligned}
$$

Let's consider the terms containing the integrals in (37). Using the solution of the stationary thermal conductivity problem (10), the temperature transformation and its derivative are written in the form $T_{\beta \alpha}(\xi)=f_{\beta \alpha} \frac{c h N \xi}{c h N h}, T_{\beta \alpha}^{\prime}(\xi)=N f_{\beta \alpha} \frac{s h N \xi}{c h N b}$. After substitution of this transform into (40), the formula for $\mathrm{w}_{\beta \alpha}^{3}(z)$ was constructed

$$
\mathrm{w}_{\beta \alpha}^{3}(z)=-\frac{\mu_{0} \rho}{4} f_{\beta \alpha} \int_{0}^{b} F(z, \xi, N) d \xi
$$

where $F(z, \xi, N)=\frac{1}{c b N h}\left(e^{-N \mid z-\xi}\left\{(N|z-\xi|+\kappa) \operatorname{shN} \xi+\mu_{*}^{-1}(z-\xi) N \operatorname{cbN} N\right\}+\frac{\phi^{(11)} \operatorname{shN} \xi-N \phi^{(12)} \operatorname{chN} \xi}{D_{N}}\right)$.

In order to find final expression for displacement (40)

$$
\mathrm{w}^{3}(x, y, z)=-\frac{\mu_{0} \rho}{4 \pi^{2}} f_{\beta \alpha} \int_{-\infty}^{\infty} \int_{0}^{\infty} \int_{0}^{h} F(z, \xi, N) e^{-i \beta y} \cos \alpha x d \beta d \alpha d \xi
$$

the representation of the function $f_{\beta \alpha}$ should be substituted into (42)

$$
\mathrm{w}^{3}(x, y, z)=-\frac{\mu_{0} \rho}{4 \pi^{2}} \int_{-\infty}^{\infty} \int_{0}^{\infty} f(\zeta, \eta) \int_{-\infty}^{\infty} \int_{0}^{\infty} \int_{0}^{b} F(z, \xi, N) e^{-i \beta y} e^{i \beta \eta} \cos \alpha x \cos \alpha \zeta d \eta d \zeta d \beta d \alpha d \xi
$$

Using formula (A3) in the Appendix 1 and (401.06., [41]), it was derived

$$
\mathrm{w}^{3}(x, y, z)=-\frac{\mu_{0} \rho \cdot C}{8 \pi} \int_{-B}^{B} \int_{0}^{A} \int_{0}^{\infty} \int_{0}^{h} t \cdot F(z, \xi, t) \cdot J_{0}^{*}(t, x, y, \zeta, \eta) d \eta d \zeta d t d \xi
$$

where the temperature is defined on a finite interval and supposed to be constant $C$.

Further, after changing the order of integration, and calculating the double integral of the Bessel function, the integral is represented in the form 


$$
\begin{aligned}
& \mathrm{w}^{3}(x, y, z)=\frac{\mu_{0} \rho \cdot C}{2 \pi} \frac{1}{N} \sum_{k=1}^{N} \frac{1}{\tau_{k} \sqrt{1-\tau_{k}^{2}}} \int_{0}^{\infty} \int_{0}^{b} F\left(t, \tau_{k}\right) \cos \left(t x \sqrt{1-\tau_{k}^{2}}\right) \cos \left(y \tau_{k}\right) \cdot \frac{F(z, \xi, t)}{t^{2}} d t d \xi \\
& \mathrm{u}^{3}(x, y, z)=\frac{\mu_{0} \rho h^{2} C}{2 \pi} \times\left(\frac{\partial}{\partial x}\right) \frac{1}{N} \sum_{k=1}^{N} \frac{1}{\tau_{k} \sqrt{1-\tau_{k}^{2}}} \int_{0}^{\infty} \int_{0}^{b} F\left(t, \tau_{k}\right) \cos \left(t \frac{x}{b} \sqrt{1-\tau_{k}^{2}}\right) \cos \left(t \frac{y}{b} \tau_{k}\right) \cdot \frac{F_{1}(z h, \xi h, t / h)}{t^{3}} d t d \xi .
\end{aligned}
$$

The expression for displacement $\mathrm{v}^{3}(x, y, z)$ can be constructed by analogy.

The expressions (38) correspond to the solution of the problem of a semi-infinite layer loading. The normal stress is constructed by the formula [20] in the form

$$
\begin{aligned}
\sigma_{x}^{A B}(x, y, z) & =\frac{2}{\pi N}\left[\sum_{k=1}^{N} \frac{\sqrt{1-\tau_{k}^{2}}}{\tau_{k}} \int_{0}^{\infty} \frac{F\left(t, \tau_{k}\right)}{D_{t}} \cdot\{(z+1) \operatorname{cht}(1-z)+(1-z) \operatorname{cht}(1+z)\} d t+\right. \\
+ & \left.\sum_{k=1}^{N} \frac{\mu_{0}^{-1} \tau_{k}^{2}-1}{\tau_{k} \sqrt{1-\tau_{k}^{2}}} \int_{0}^{\infty} \frac{F\left(t, \tau_{k}\right)}{D_{t} \cdot t} \cdot\{\operatorname{sht}(1+z)+\operatorname{sht}(1-z)\} d t\right]
\end{aligned}
$$

The final solution of the initially stated uncoupled thermoelasticity problem for a semi-infinite layer with its proper weight was derived in the form

$$
\begin{aligned}
& \sigma_{x}^{*}(x, y, z)=\sigma_{x}^{T+A B}(x, y, z)+2 \mu(1-\mu) q_{z} h(1-z), \\
& \sigma_{x}^{T+A B}(x, y, z)=\sigma_{x}^{A B}(x, y, z) \cdot(1-G \rho C)- \\
& \quad-\frac{2 G \rho C \mu_{0}^{2}}{\pi N}\left[\sum_{k=1}^{N} \frac{1-\mu-\mu_{0}^{-1} \tau_{k}^{2}}{\tau_{k} \sqrt{1-\tau_{k}^{2}}} \int_{0}^{\infty} \int_{0}^{\infty} \frac{F\left(t, \tau_{k}\right)}{c b t} F_{1} d \xi d t+\sum_{k=1}^{N} \frac{1}{\tau_{k} \sqrt{1-\tau_{k}^{2}}} \int_{0}^{1} \int_{0}^{\infty} \frac{F\left(t, \tau_{k}\right)}{c b t} F_{2} d \xi d t\right],
\end{aligned}
$$

where the functions $F_{1}, F_{2}$ are defined in Appendix 5, $\rho=\alpha \mu_{*}, \alpha$ is a coefficient of linear expansion, $D_{t}=\operatorname{sh} 2 t+2 t$, $F\left(t, \tau_{k}\right)=\sin \left(t A_{b} \sqrt{1-\tau_{k}^{2}}\right) \sin \left(t B_{b} \tau_{k}\right) \cos \left(t x_{b} \sqrt{1-\tau_{k}^{2}}\right) \cos \left(t y_{b} \tau_{k}\right)$.

\section{DISCUSSION AND NUMERICAL RESULTS}

$\mathrm{D}$

uring the calculations, two types of materials were selected: Copper $-\mu=1 / 3, G=44.7 \mathrm{GPa}, \alpha=16.5 \cdot 10^{-6}$ $1 / \mathrm{C}^{0}, q_{z}=0.00896 \mathrm{~kg} / \mathrm{M}^{3} ;$ Glass $-\mu=1 / 4, G=26.2 \mathrm{GPa}, \alpha=6 \cdot 10^{-6} 1 / \mathrm{C}^{0}, q_{z}=0.00119 \mathrm{~kg} / \mathrm{m}^{3}$; the layer's thickness $h=1 \mathrm{M}$;

In all diagrams and tables, the units of measurement for stress are $P a$;

The investigation of the influence of the load area shape on the stress was carried out for the case of unit temperature. For glass, in Figures 2, 3 the distributions of normal stress $\sigma_{x}^{A B}, \sigma_{x}^{A B+T}$ along the lateral wall of the layer for $0<z<b$ depending on the shapes of the distributed load section and the temperature are represented. Here $\sigma_{x}^{A B}$ indicates the normal stress caused by mechanical loading, distributed over the site $x \in[0, A], y \in[-B, B], \sigma_{x}^{A B+T}$ - normal stress under the action of distributed load and temperature influence.

The case $B=2 A$ corresponds to the distribution of compression loading on the layer's face $z=b$ along the rectangle, elongated along the axis $y ; B=A / 4$ - along a rectangle elongated along the axis $x ; B=A / 2$ - quadratic spread.

These graphs correspond to the case for glass. The stress graphs for copper, where $\mu=1 / 3$, are similar, but with larger absolute values. So, they are not shown here.

As it can be seen from the graphs, in the case of a shape $B=A / 2$, maximal compressing stress is observed. In the case 
of size $B=2 A$ the stress has minimal values and the separation of the layer from the side wall takes place. Stretching stresses are greater for the case of size $B=2 A$ in comparison with the form $B=A / 2$. Taking into account the temperature influence, one can note increasing compressing stress values and reducing tensile stress values.

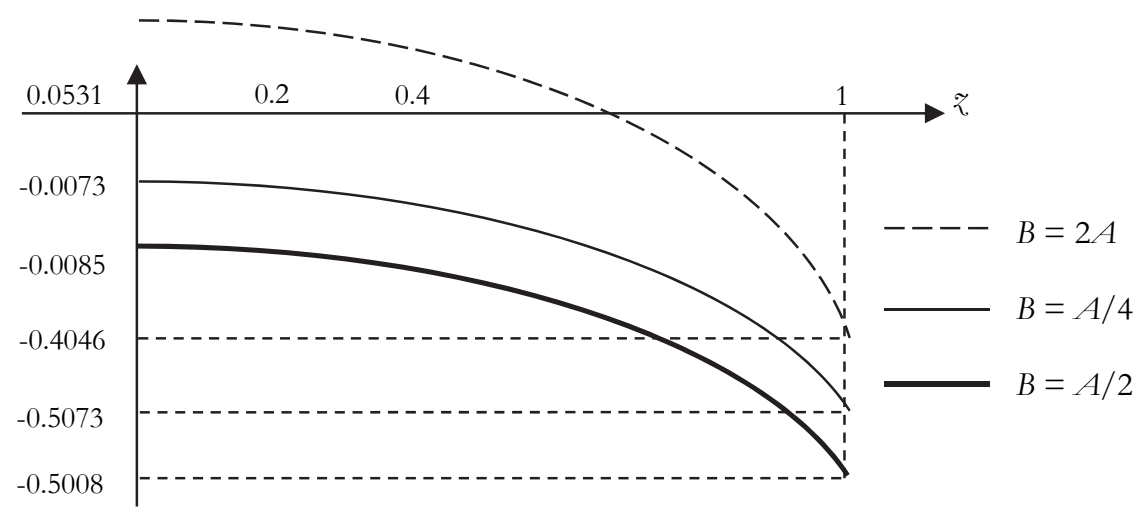

Figure 2: Distribution of normal stress $\sigma_{x}^{A B}(0,0, z)$

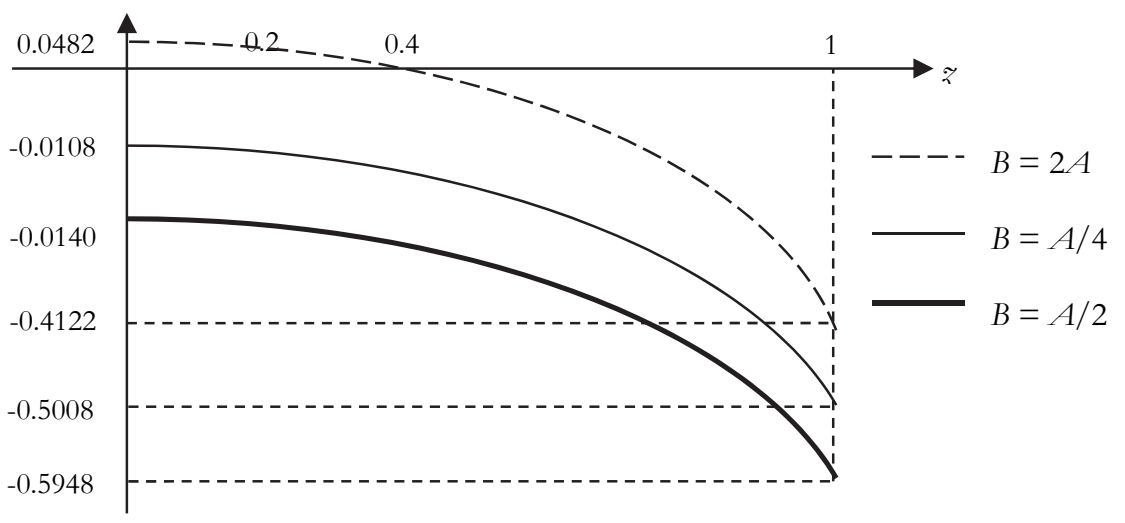

Figure 3: Distribution of normal stress $\sigma_{x}^{A B+T}(0,0, z)$

It was also important to establish for which materials and loading sites a delamination of the side wall of the layer occurs. It was found that the separation of a semi-infinite layer from the side wall is not observed when the ratio $P / C \geq 12$, where $P$ - the dimensionless intensity of constant mechanical loading, $C$ - the dimensionless temperature. The side edge of the semi-infinite layer is detached for Poisson's ratio values $0<\mu<0.33$ at the size $B=2 A$ and for values $0<\mu<0.23$ at the size $B=A / 2$ (Fig. 4).

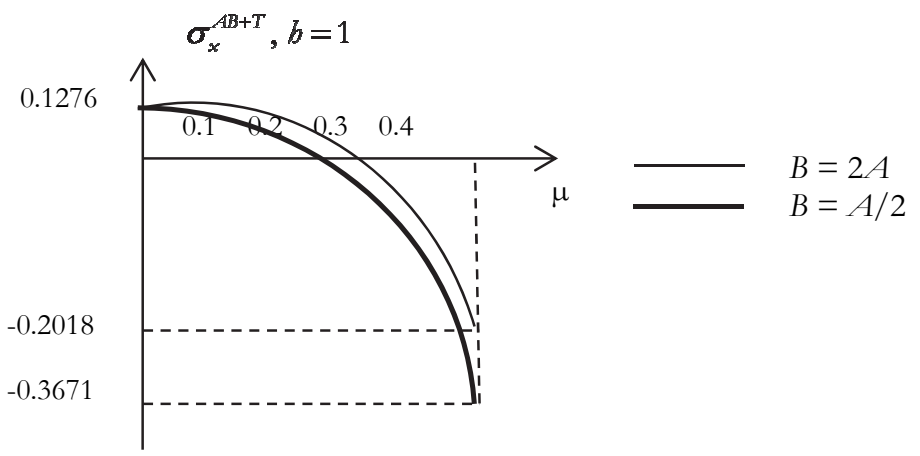

Figure 4: Distribution of normal stress depends on Poisson's ratio values 
The layer separation was observed in different cases of Poisson's ratio values. It was established which values of coefficient lead to the delamination of the lateral face of the semi-infinite layer. In these cases, the linearity of the problem is violated and the statement of the problem is not correct.

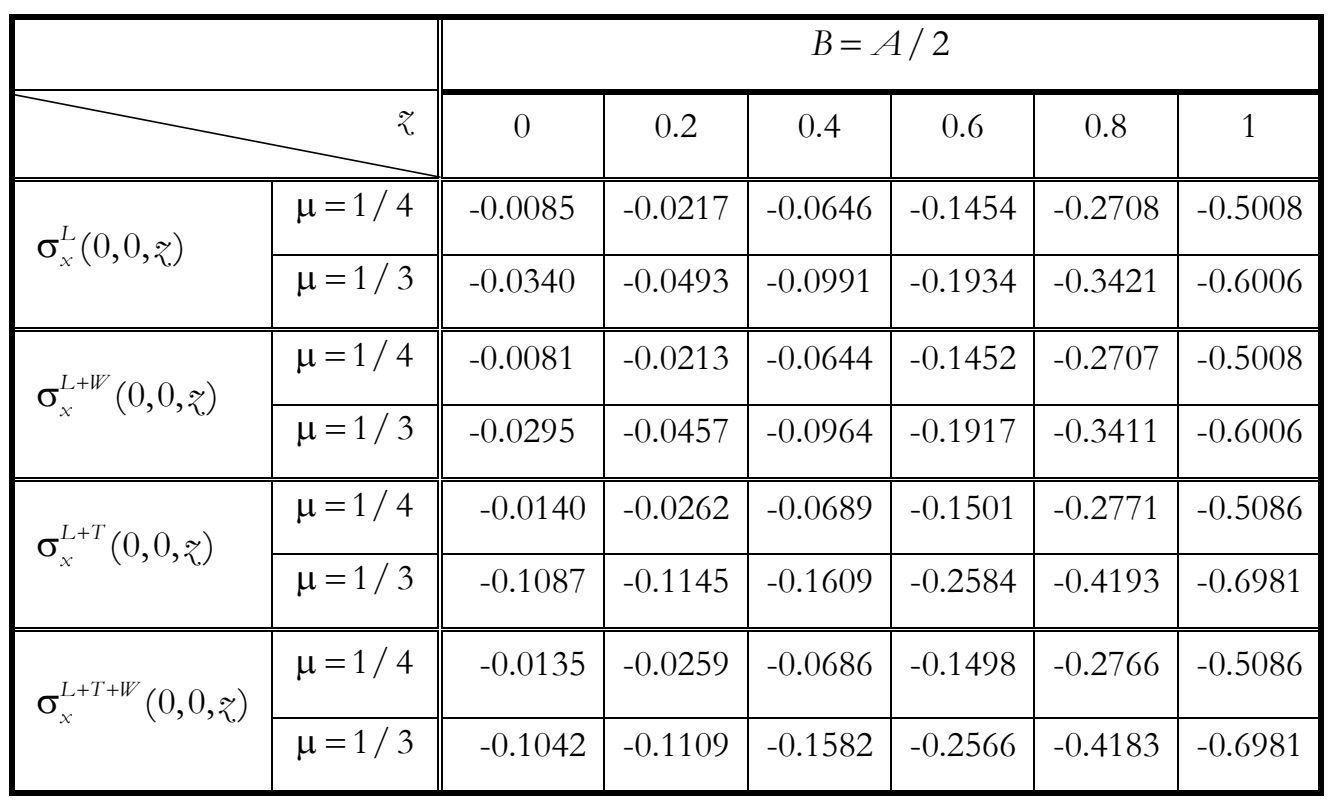

Table 1: Normal stress along the axis ₹

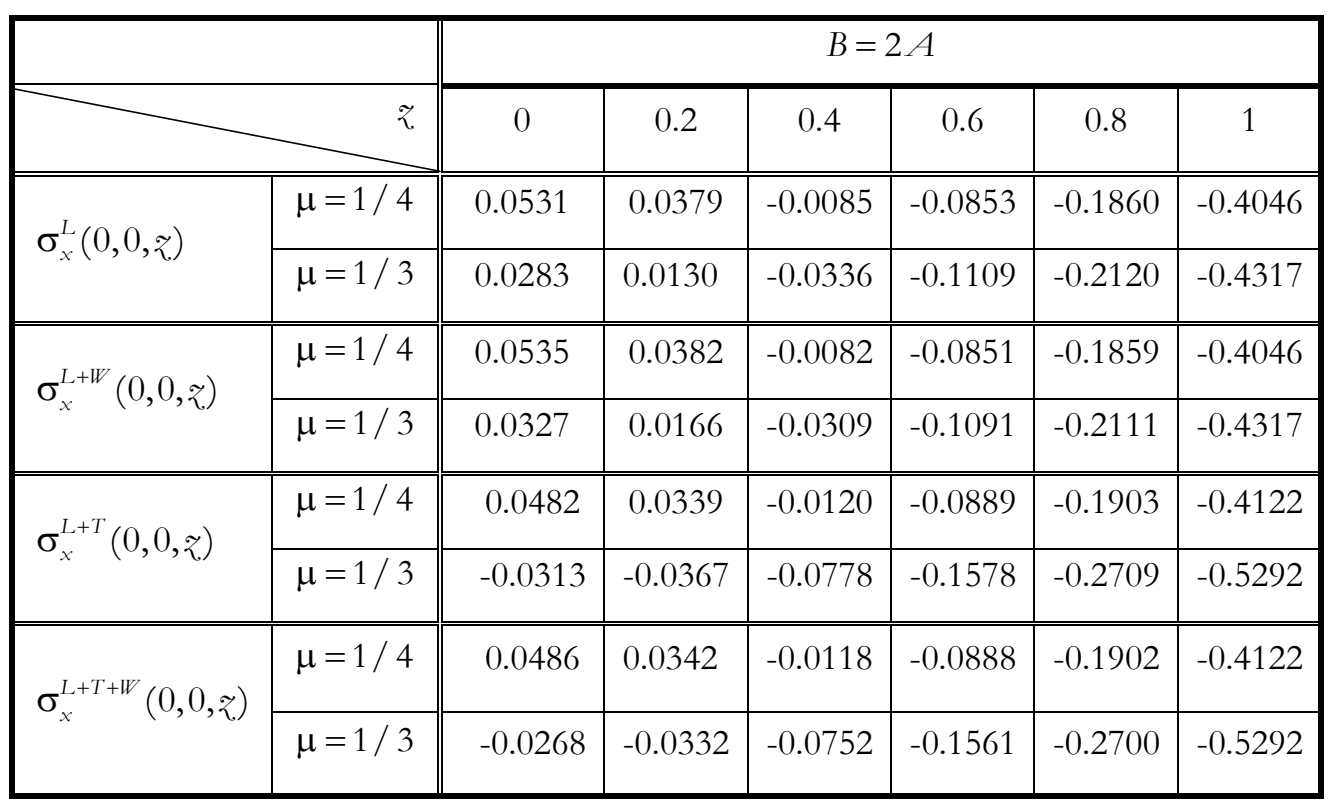

Table 2: Normal stress along the axis z

The values of the normal stress are given in the tables (1)-(3) for the following parameters: the thickness of the layer $b=1$ and unit temperature. They are investigated depending on the form of the load and temperature distribution. For convenience, the following notations are introduced:

$$
\sigma_{x}^{L}-\text { the stress that occurs under the action of a compressive load; }
$$


$\sigma_{x}^{L+W}$ - the stress that occurs under the action of a compressive load with regard to the proper weight of the layer;

$\sigma_{x}^{L+T}$ - the stress that occurs under the action of a compressive load with a distributed temperature;

$\sigma_{x}^{L+T+W}$ - the stress that occurs under the action of a compressive load with a distributed temperature with regard to the proper weight of the layer;

\begin{tabular}{|r|r||c|c|c|c|c|c|}
\hline \multicolumn{2}{|c||}{$z$} & 0 & 0.2 & 0.4 & 0.6 & 0.8 & 1 \\
\hline \hline \multirow{3}{*}{$\sigma_{x}^{L}(0,0, z)$} & $\mu=1 / 4$ & -0.0073 & -0.0156 & -0.0439 & -0.1042 & -0.2291 & -0.5737 \\
\cline { 2 - 8 } & $\mu=1 / 3$ & -0.0220 & -0.0318 & -0.0656 & -0.1389 & -0.2952 & -0.7276 \\
\hline \hline \multirow{3}{*}{$\sigma_{x}^{L+W}(0,0, z)$} & $\mu=1 / 4$ & -0.0069 & -0.0153 & -0.0436 & -0.1041 & -0.2290 & -0.5737 \\
\cline { 2 - 8 } & $\mu=1 / 3$ & -0.0295 & -0.0282 & -0.0629 & -0.1372 & -0.2943 & -0.7276 \\
\hline \hline \multirow{3}{*}{$\sigma_{x}^{L+T}(0,0, z)$} & $\mu=1 / 4$ & -0.0108 & -0.0186 & -0.0471 & -0.1501 & -0.2771 & -0.5948 \\
\cline { 2 - 8 } & $\mu=1 / 3$ & -0.0706 & -0.0783 & -0.1193 & -0.2135 & -0.4141 & -0.9970 \\
\hline \hline \multirow{3}{*}{$\sigma_{x}^{L+T+W}(0,0, z)$} & $\mu=1 / 4$ & -0.0104 & -0.0182 & -0.0468 & -0.1085 & -0.2368 & -0.5948 \\
\cline { 2 - 8 } & $\mu=1 / 3$ & -0.0661 & -0.0747 & -0.1166 & -0.2117 & -0.4132 & -0.9971 \\
\hline
\end{tabular}

Table 3: Normal stress along the axis ₹

It can be seen from analysis of these tables that the separation of the layer from the rigid wall arises in the case of the size $B=2 A$. This case corresponds to the distribution of temperature and loading elongated along the $y$ axis by a rectangular spot. Here the stresses are higher for the case of Poisson's value $\mu=1 / 4$.

The influence of loading begins to have a significant effect on the absolute value of the stress at about $2 / 3$ of the layer $b$ height. This pattern can be traced for any shape of the loading area and Poisson's ratio.

The investigation of the loading spot's shape was provided with the aim to establish what ratio of length and width causes the maximal value of normal stress. It was established that maximal stress, equal to -0.7276 , arises on the side wall of the layer as a result of the mutual action of the distributed load and proper weight of the layer. It has place in the case when the load is distributed along a rectangle elongated along the axis $x$, when $\mu=1 / 3$.

The maximal value, equal to -0.9971 , the stress reaches when $B=A / 4$, when the load, temperature influence and proper weight are taken into account, i.e. the stress practically becomes equal to the acting load. In all cases it is clear that taking into account the proper weight of the layer reduces the values of the compressive normal stresses on the side wall. The combined influence of temperature and external load increases the stress on the lateral face of the layer compared with the case when only the external mechanical action is taken into account.

\section{ConClusions}

7 he proposed new method, based on the Green's matrix function apparatus, made it possible to obtain the exact solution of the uncoupled thermoelasticity problem for a semi-infinite layer taking into account its proper weight. The engineering computational formulas for the displacements and stress of the layer were obtained and the 
mechanical characteristics that arise at certain ratios of the distribution spot, where mechanical loading and temperature are acting, were revealed. The resulting formulas can be used as models for estimating the validity of the new approximate numerical methods to solve mechanical problems.

The analysis of the calculations establishes the geometrical parameters of the loading spots shape sections, when the effect of the layer's edge separation from the wall is observed. It was found that the appearance of the tensile stress is sufficiently dependent on each material from the shape of the loading spot. It was also revealed at what values of the Poisson's ratio the separation of the layer's wall is observed and the problem's statement becomes incorrect. It was determined that taking into account the layer's proper weight reduces the stress values. The presence of temperature load increases the value of stress in comparison with the case, when only a mechanical load is applied. The analyses of stress for different shapes of the loading spots as with, so and without layer proper weight indicates that maximal the value of the stress is observed when the shape of loading spot is quadratic.

It should be emphasized that the frictionless contact conditions at the layer's edge made it possible to obtain an exact solution of the problem. In the case when the edge is rigidly fixed to the wall, the initial problem is reduced to an integral singular equation. The developed methodology allows a number of extensions. This approach can be used to solve a wider class of problems, when different boundary conditions are fulfilled on the faces of the layer or when the load is nonstationary one. Analysis of some cases of steady-state loading is already in progress.

\section{REFERENCES}

[1] Eubanks, R. A. and Sternberg, E. (1956). On the completeness of the Boussinesq-Papkovitch stress functions, J. Rat. Mech. And Anal. 5, pp. 735-746.

[2] Papkovitch, P. F. (1932). A representation of the general integral of the basic differential equations of elasticity theory in terms of harmonic functions, Izv. Akad. Nauk SSSR. Otd. Mat. Est. Nauk., 10. pp. 1425-1435.

[3] Neuber, H. (1973). Kerbspanungslehre, Berlin, Springer.

[4] Kolosov, G. V. (1935). Application of Complex Diagrams and the Theory of Functions of Complex Varable to Elasticity Theory [in Russian], Leningrad-Moscow, ONTI.

[5] Muskhelishvili, N. I. (1953). Some Basic Problems of Mathematical Theory of Elasticity, Groningen, Noordhoff.

[6] Polozhii, G. N. (1963). On the boundary-value problems of axisymmetric elasticity theory. The method of $p-$ analytic functions of complex variable, Ukr. Mat. Zh., 15 (1), pp. 25-45.

[7] Kupradze, V. D., Gegeliya, T. G., Basheleishvili, M. O. and Burchuladze, T. V. (1976). Three- Diminsional Problems of Mathematical Elasticity Theory and Thermoelasticity [in Russian], Moscow, Nauka.

[8] Uflyand, Ya. S. (1968). Integral Transformations in Problems of Elasticity Theory [in Russian], Leningrad, Nauka.

[9] Ulitko, A. F. (1979). The Method of Vector Eigenfunctions in Spatial Problems of Elasticity Theory [in Russian], Kiev, Naukova Dumka.

[10] Kit, H. S. (2008). Problems of stationary heat conduction and thermoelasticity for a body with heat release on circular domain (crack), Mat. Met. Fis.-Mech. Polya, 51 (4), pp. 120-128; English translation: (2010). J. Math. Sci. 167 (2), pp. 141-153.

[11] Popov, G. Ya. (2003). New Transforms for the Resolving Equations in Elastic Theory and New Integral Transforms, with Applications to Boundary Value Problems of Mechanics, Prikl. Mekh., 39 (12), pp. 46-73. English translation: (2003). Int. Appl. Mech., 39 (12), pp. 1400-1424.

[12] Vaisfeld, N. D., Popov, G. Ya. (2009). Mixed boundary value problem of elasticity for a quarter space, Mech. Solids. 44: 712. DOI: $10.3103 /$ S0025654409050082

Original Russian Text: (2009). Izvestiya Akademii Nauk. Mekhanika Tverdogo Tela, 5, pp. 68-89.

[13] Takhar, H. S., Chamkha, A. J., Nath, G. (1999). Unsteady flow and heat transfer on a semi-infinite flat plate with an aligned magnetic field, Int. J. Engineering Sci. 37, pp. 1723-1736.

[14] Tokovyy, Y., Ma, C.-C. (2015). An analytical solution to the three-dimensional problem on elastic equilibrium of an exponentially-inhomogeneous layer, J. Mech. 31 (5), pp. 545-555.

[15] Vaysfel'd, N., Zhuravlova, Z. (2015). On one new approach to the solving of an elasticity mixed plane problem for the semi-strip, Acta Mechanica, 226: 4159. DOI: 10.1007/s00707-015-1452-x

[16] Fesenko, A. A. (2015). Mixed problems of stationary heat conduction and elasticity theory for a semiinfinite layer, J. Math. Sci., 205 (5), pp. 706-718. DOI: 10.1007/s10958-015-2277-9

[17] Haji-Sheikh, A., Donald, E. A., Beck, J. V. (2009). Temperature field in a moving semi-infinite region with a prescribed wall heat flux, Int. J. Heat and Mass Transfer, 52 (7), pp. 2092-2101. 
[18] Kit, H. S., Mykhas' kiv, V. V., Khai, M. V. (1996). The Method of Potentials in Three-Dimensional Static and Dynamical Problems of the Theory of Cracks, Materials Science, 32 (1), pp. 14-24.

[19] Matysiak, S. J., Perkowski, D. M. (2010). On heat conduction in a semi-infinite laminated layer. Comparative results for two approaches, Int. Communications in Heat and Mass Transfer, 37, pp. 343-349.

[20] Nowacki, W. (1957). A dynamical problem of thermoelasticity, Archiwum Mechaniki Stosowanej, 9 (3), pp. 325-334.

[21] Rabinovich, A., Dagan, G., Miloh T. (2012). Heat conduction in a semi-infinite medium with a spherical inhomogeneity and time-periodic boundary temperature, Int. J. Heat and Mass Transfer, 55, pp. 618-628.

[22] Tokovyy, Y., Ma, C.-C. (2013). Three-dimensional temperature and thermal stress analysis of an inhomogeneous layer, J. Therm. Stresses, 36 (8), pp. 790-808.

[23] Kulchytsky-Zhyhailo, R., Matysiak, S. J. \& Perkowski, D. M. (2007). On displacements and stresses in a semi-infinite laminated layer: comparative results, Meccanica, 42: 117. DOI: 10.1007/s11012-006-9026-6

[24] Chen, Y.Z. (2016). Numerical solution for thermal confocal elliptic dissimilar layers in plane elasticity, Acta Mechanica, 227: 2233-2244. DOI: 10.1007/s00707-016-1626-1

[25] Menshykov, O., Menshykova, M. and Vaysfeld, N. (2017). Exact analytical solution for a pie-shaped wedge thick plate under oscillating load, Acta Mechanica, 228 (12), pp. 4435-4450. DOI: 10.1007/s00707-017-1938-9

[26] Vorovich, I. I. and Babeshko, V. A. (1979). Dynamical Mixed Problems of the Elasticity Theory for the No Classical Areas [in Russian], Moscow, Nauka.

[27] Taein, Yeo and Barber, J. R. (1996). Finite element analysis of the stability of static thermoelastic contact, J. Thermal Stresses, 19 (2), pp. 169-184 DOI: 10.1080/01495739608946168

[28] Popov, G. Ya., Kebli, B. (2012). Exact solution of the mixed boundary value elasticity problem for an infinite wedgeshaped plate with regard for its proper weight, J. Math. Sci., 187 (6), pp. 758-771.

[29] Popov, G. Ya., Protserov, Yu. S. (2016). Axisymmetric Problem for an Elastic Cylinder of Finite Length with Fixed Lateral Surface with Regard for its Weight, J. Math. Sci., 212 (1), pp. 67-82.

[30] Bateni, M. and Eslami, M. R. (2018). Thermally nonlinear generalized thermoelasticity: a note on the thermal boundary conditions, Acta Mechanica, 229, pp. 807-826. DOI: 10.1007/s00707-017-2001-6

[31] Bostani, M. and Karami Mohammadi, A. (2018). Thermoelastic damping in microbeam resonators based on modified strain gradient elasticity and generalized thermoelasticity theories, Acta Mechanica, 229, pp. 173-192. DOI: 10.1007/s00707-017-1950-0

[32] Shishesaz, M., Hosseini, M., Naderan Tahan, K. et al. (2017). Analysis of functionally graded nanodisks under thermoelastic loading based on the strain gradient theory, Acta Mechanica, 228, pp. 4141-4168. DOI: 10.1007/s00707-017-1939-8

[33] Ebrahimi, H. Z. and Haghighi, M. R. G. (2016). Estimation of heat flux and mechanical loads on laminated functionally graded plate, Acta Mechanica, 227, pp. 2075-2097. DOI: 10.1007/s00707-016-1596-3

[34] Kugler, S., Fotiu, P.A. and Murin. (2016). Thermo-elasticity in shell structures made of functionally graded materials, Acta Mechanica, 227: 1307. DOI: 10.1007/s00707-015-1550-9

[35] Senthil, S. Vel and Batra, R. C. (2002). Exact Solution for Thermoelastic Deformations of Functionally Graded Thick Rectangular Plates, AIAA Journal, 40 (7), pp. 1421-1433. DOI: 10.2514/2.1805

[36] Babeshko, V., Evdokimova, O., Babeshko, O. (2017). Action of the semi-infinite plates on the elastic layer, Procedia IUTAM, 20, pp. 50-55. DOI: 10.1016/j.piutam.2017.03.007

[37] Marsavina, L., Tomlinson, R. A. (2014). Frattura ed Integrità Strutturale, 8 (27), pp. 13-20. DOI: 10.3221/IGFESIS.27.02

[38] Pitarresi, G., Scalici, T., Catalanotti, G. (2018). Thermoelastic Stress Analysis of modified Transverse Cut Tensile composite specimens under pure Mode II fatigue delamination, Procedia Structural Integrity, 8, pp. 474-485. DOI: 10.1016/j.prostr.2017.12.047

[39] Popov, G. Ya., Abdimanov, S. A. and Ephimov, V. V. (1999). Green's Functions and Matrices of One-Dimensional Boundary Value Problems [in Russian], Almaty, Rauan.

[40] Popov, G. Ya. (1982). The Elastic Stress Concentration Around Dies, Cuts, Thin Inclusions and Reinforcements [in Russian], Moscow, Nauka.

[41] Gradshtein, I. S. and Rygik, I. M. (1980). Tables of Integrals, Series and Products, New York, Acad. Press.

[42] Bateman, H. and Erdelyi, A. (1954). Higher Transcendental Functions, New York, McGraw-Hill. 


\section{APPENDIX 1. DERIVING THE FORMULA (12) FOR THE TEMPERATURE FUNCTION}

The corresponding formula for $f_{\alpha \beta}, f_{\alpha \beta}=\int_{-\infty}^{\infty} \int_{0}^{\infty} f(x, y) \cos \alpha x \cdot e^{i \beta y} d y d x$ is substituted at (11) and the order of integration operators is changed at (11). To calculate the integrals exactly, the formula (1.314(3), [41]) is used

$$
T(x, y, z)=\frac{1}{\pi^{2}} \int_{0}^{\infty} \int_{-\infty}^{\infty}\left(\int_{0}^{\infty} \int_{-\infty}^{\infty} f(\xi, \eta) \cos \alpha \xi \cdot e^{i \beta \eta} d \xi d \eta\right) \frac{c b N z}{c b N b} \cdot \cos \alpha x \cdot e^{-i \beta y} d \alpha d \beta
$$

Let's consider the internal integrals at formula (A1). One must use the fact that function $f(\xi, \eta)$ is the even one and to use Euler's formula. After some additional transforms the solution of the boundary value problem (7-9) is written as

$$
T(x, y, z)=\frac{1}{4 \pi^{2}} \int_{0}^{\infty} \int_{-\infty}^{\infty} f(\xi, \eta)\left(\int_{-\infty}^{\infty} \int_{-\infty}^{\infty} \frac{c h N z}{c h N h}\left[e^{-i \alpha(x+\xi)}+e^{-i \alpha(x-\xi)}\right] e^{-i \beta(y-\eta)} d \alpha d \beta\right) d \xi d \eta
$$

One should note that value $N$ at the solution (A2) is defined as $N^{2}=\alpha^{2}+\beta^{2}$. It gives opportunity to simplify the expression (A2) with the formula [40]

$$
\frac{1}{2 \pi} \int_{-\infty}^{\infty} \int_{-\infty}^{\infty} F\left(\sqrt{\alpha^{2}+\beta^{2}}\right) e^{-i \alpha x-i \beta y} d \alpha d \beta=\int_{0}^{\infty} t F(t) J_{0}\left(t \sqrt{x^{2}+y^{2}}\right) d t
$$

where $J_{0}(t)$ is a Bessel's function of the first kind.

With regard of formula (A3), the solution (A2) will be transformed

$$
T(x, y, z)=\frac{1}{2 \pi} \int_{0}^{\infty} \int_{-\infty}^{\infty} f(\xi, \eta)\left(\int_{0}^{\infty} t \frac{c b t z}{c b t h} \cdot J_{0}^{*}(t, x, y, \xi, \eta) d t\right) d \xi d \eta
$$

where $J_{0}^{*}(t, x, y, a, b)=J_{0}\left(t \sqrt{(x-a)^{2}+(y-b)^{2}}\right)+J_{0}\left(t \sqrt{(x+a)^{2}+(y-b)^{2}}\right)$.

If at the boundary condition (9) one takes $f_{\alpha \beta} \equiv C, C$ is constant, then exact solution of boundary value problem (7-9) will be obtained

$$
T(x, y, z)=\frac{C}{2 \pi} \int_{0}^{A} \int_{-B}^{B}\left(\int_{0}^{\infty} t \frac{c b t z}{c b t h} \cdot J_{0}^{*}(t, x, y, \xi, \eta) d t\right) d \xi d \eta
$$

Let's simplify the internal integral at the equality (A5), using the known integral representation of Bessel function [42]

$$
J_{0}\left(t \sqrt{(x \pm \xi)^{2}+(y-\eta)^{2}}\right)=\frac{2}{\pi} \int_{0}^{\frac{\pi}{2}} \cos [t \cos \psi(x \pm \xi)] \cos [t \sin \psi(y-\eta)] d \psi
$$

After some elementary transformations one obtains

$$
T(x, y, z)=C \frac{4 A B}{\pi^{2}} \int_{0}^{\frac{\pi}{2} \infty} \int_{0}^{\infty} t \frac{c b t z}{c h t h} S_{t}^{A, B}(\psi) \cos (t x \cos \psi) \cos (t y \sin \psi) d \psi d t
$$


here

$$
S_{t}^{A, B}(\psi)=(t A \cos \psi)^{-1} \sin (t A \cos \psi)(t B \sin \psi)^{-1} \sin (t B \sin \psi)
$$

As it may be seen function $S_{t}^{A, B}(\psi)$ is continuously differentiated function with regard of variable $\psi$. This function is even also, so after changing the variable

$$
\sin \psi=\tau
$$

one can obtain the correspondence

$$
J_{t}^{A, B}(x, y)=\frac{2 A B}{\pi^{2}} \int_{-1}^{1} F_{t, \tau}^{A, B}(x, y) \frac{d \tau}{\sqrt{1-\tau^{2}}}
$$

where $F_{t, \tau}^{A, B}(x, y)=\frac{\sin \left(t A \sqrt{1-\tau^{2}}\right)}{t A \sqrt{1-\tau^{2}}} \frac{\sin (t B \tau)}{t B \tau} \cos \left(t x \sqrt{1-\tau^{2}}\right) \cos (t y \tau)$.

Based on the quadrature formula of the highest degree of accuracy, one can obtain for (A6)

$$
J_{t}^{A, B}(x, y)=\frac{2 A B}{\pi} \frac{1}{N} \sum_{k=1}^{N} F_{t, \tau_{k}^{(N)}}^{A, B}(x, y), \tau_{k}^{(N)}=\cos \frac{2 k-1}{2 N} \pi, \quad k=\overline{1, N}
$$

$\tau_{k}^{(N)}$ are zeros of the Chebyshov polynomials of the first kind. So, after substitution we get

$$
T(x, y, z)=\frac{2 C}{\pi N} \sum_{k=1}^{N} \int_{0}^{\infty} \frac{c b(t z)}{c h(b t)} \frac{\sin \left(t A \sqrt{1-\tau_{k}^{2}}\right) \sin \left(t B \tau_{k}\right)}{t A \sqrt{1-\tau_{k}^{2}} B \tau_{k}} \cos \left(t x \sqrt{1-\tau_{k}^{2}}\right) \cos \left(t y \tau_{k}\right) d t
$$

\section{APPENDIX 2. DERIVING THE FORMULA (13)}

For the analysis of the reliability of the calculations, we perform a test based on the obtained formula (12) for the fulfillment of the boundary condition of the problem. To this end, we consider the temperature (12) in the corner point of the layer $x=0, y=0, z=b$

$$
T(0,0, h)=\frac{2 C}{\pi N} \sum_{k=1}^{N} \int_{0}^{\infty} t F_{t, \tau}^{A, B}(0,0) d t=\frac{2 C}{\pi N} \sum_{k=1}^{N} \int_{0}^{\infty} \frac{\sin \left(t A \sqrt{1-\tau_{k}^{2}}\right) \sin \left(t B \tau_{k}\right)}{t A \sqrt{1-\tau_{k}^{2}} B \tau_{k}} d t
$$

Using the formula (3.741(1), [41])

$$
\int_{0}^{\infty} \frac{\sin m x \sin n x}{x} d x=\frac{1}{4} \ln \left(\frac{m+n}{m-n}\right)^{2}, m>0, n>0, m \neq n
$$

gives

$$
T(0,0, b)=\frac{C}{2 \pi N} \sum_{k=1}^{N} \frac{1}{\tau_{k} \sqrt{1-\tau_{k}^{2}}} \ln \left(\frac{\sqrt{1-\tau_{k}^{2}}+(B / A) \tau_{k}}{\sqrt{1-\tau_{k}^{2}}-(B / A) \tau_{k}}\right)^{2}
$$


We note that when $m=0, n \neq 0$ or $n=0, m \neq 0$ the formula (A8) is not contradictory, it is also possible $n<0 \quad(m<0)$. To calculate with this formula, an essential condition is the requirement $m \neq n$, i.e $A \sqrt{1-\tau_{k}^{2}} \neq B \tau_{k}$. If $B / A=1$, then, to be definite, it is necessary that the condition $\sqrt{1-\tau^{2}}-\tau \neq 0$, or based on the replacement $\sin \psi=\tau: \cos \psi \neq \sin \psi$, i.e $\psi \neq \pi / 4$ or $\tau \neq \pm 1 / \sqrt{2}$. Let's consider the case $A \neq B$. Taking into account formula (A9), we get

$$
T(0,0, h)=\frac{C}{2 \pi N} \sum_{k=1}^{N} \frac{1}{\tau_{k} \sqrt{1-\tau_{k}^{2}}} \ln \left(\frac{\sqrt{1-\tau_{k}^{2}}+(B / A) \tau_{k}}{\sqrt{1-\tau_{k}^{2}}-(B / A) \tau_{k}}\right)^{2}
$$

If $N-$ is an even number, then proceeding from the properties of the logarithm, the expression for the temperature (A10) takes the form

$$
T(0,0, h)=\frac{C}{\pi N} \sum_{k=1}^{N / 2} \frac{1}{\tau_{k} \sqrt{1-\tau_{k}^{2}}} \ln \left(\frac{\sqrt{1-\tau_{k}^{2}}+(B / A) \tau_{k}}{\sqrt{1-\tau_{k}^{2}}-(B / A) \tau_{k}}\right)^{2} .
$$

Here we use the property of zeros of the Chebyshev polynomial: $\tau_{N}=-\tau_{1}, \tau_{N-1}=-\tau_{2}, \ldots$, i.e. there are exactly $N$ roots, arranged symmetrically in the gap $(-1,1)$. It can be noted that when $N-$ is odd, there will be a root $\tau_{(N+1) / 2}=0$, which leads to a singularity in formula (A10). For this case we consider the passage to the limit $\varepsilon \rightarrow 0$ $\tau_{k}=\tau_{k}+\varepsilon, \tau_{k}=0, k=(N+1) / 2$. Using the Lopital rule, we have

$$
T(0,0, h)=\frac{C}{\pi N}\left(2 \frac{B}{A}+\frac{1}{2} \sum_{\substack{k=1 \\ k \neq(N+1) / 2}}^{N} \frac{1}{\tau_{k} \sqrt{1-\tau_{k}^{2}}} \ln \left(\frac{\sqrt{1-\tau_{k}^{2}}+(B / A) \tau_{k}}{\sqrt{1-\tau_{k}^{2}}-(B / A) \tau_{k}}\right)^{2}\right.
$$

Let us consider three cases of local temperature distribution over sections of different sizes:

1) $B / A=1 / 2$;2) $B / A=2$; 3) $B / A=1 / 4$.

For the case when the number of nodes $N$ in the formula (A10) was chosen equal to $N=70$ and the constant of the temperature $C=1$, for three cases, the temperature at the corner point of the layer was equal to:

1) $T(0,0, h)=0.999445,2) T(0,0, h)=0.999444,3) T(0,0, h)=0.998545$.

The reliability of the results obtained was verified on the basis of formula (3.364(2), [41]).

We can justify the result directly, considering the boundary condition (9) of the problem. Proceeding from the applied integral transformations, one can find the transformation of the given function

$$
\begin{aligned}
& f_{\alpha}(y)=\int_{0}^{\infty} f(x, y) \cos \alpha x d x=C \cdot \int_{0}^{A} \cos \alpha x d x=C \cdot \frac{\sin \alpha A}{\alpha}, \\
& f_{\alpha \beta}=\int_{-\infty}^{\infty} f_{\alpha}(y) e^{i \beta y} d y=C \cdot \frac{\sin \alpha A}{\alpha} \int_{-B}^{B} e^{i \beta y} d y=C \cdot \frac{\sin \alpha A}{\alpha} \cdot \frac{2\left(e^{i \beta B}-e^{-i \beta B}\right)}{2 i \beta}=2 C \cdot \frac{\sin \alpha A}{\alpha} \cdot \frac{\sin \beta B}{\beta} .
\end{aligned}
$$

We substitute the resulting transform into formula (12), and find the value at the point $(0,0, h)$

$$
T(0,0, h)=\frac{2 C}{\pi^{2}} \int_{0}^{\infty} \int_{-\infty}^{\infty} \frac{\sin \alpha A}{\alpha} \cdot \frac{\sin \beta B}{\beta} d \alpha d \beta=\frac{4 C}{\pi^{2}} \cdot \frac{\pi}{2} \cdot \frac{\pi}{2}=C
$$


Here we used the parity of the integrand with respect to the variable $\beta$ and formula (3.721(1), [41]). Thus, the numerically obtained results are justified analytically, by the relation

$$
\frac{C}{2 \pi N} \sum_{k=1}^{N} \frac{1}{\tau_{k} \sqrt{1-\tau_{k}^{2}}} \ln \left(\frac{\sqrt{1-\tau_{k}^{2}}+\frac{B}{A} \tau_{k}}{\sqrt{1-\tau_{k}^{2}}-\frac{B}{A} \tau_{k}}\right)^{2}=C .
$$

\section{APPENDIX 3. DERIVATIVES OF THE SOLUTIONS $\mathbf{Y}_{\mp}(z)$ AND CONSTANT MATRICES}

$$
\begin{aligned}
& \mathbf{Y}_{-}^{\prime}(z)=\mu_{0} e^{-N z}\left(\begin{array}{cc}
N\left(N z+2 \mu_{0}^{-1}\right) & \mu_{*}^{-1}(-N z+1) \\
N^{2}(N z-1) & \mu_{*}^{-1} N\left(-N z+2 \mu_{1}^{-1}\right)
\end{array}\right), \mathbf{Y}_{+}^{\prime}(z)=\mu_{0} e^{N z}\left(\begin{array}{cc}
N\left(-N z+2 \mu_{0}^{-1}\right) & -\mu_{*}^{-1}(N z+1) \\
N^{2}\left(N_{z}+1\right) & \mu_{*}^{-1} N\left(N z+2 \mu_{1}^{-1}\right)
\end{array}\right) \\
& \mathbf{C}_{0}^{1}=\left(U_{0}\left[\mathbf{Y}_{+}\right]-U_{0}\left[\mathbf{Y}_{-}\right] \cdot\left(U_{1}\left[\mathbf{Y}_{-}\right]\right)^{-1} \cdot U_{1}\left[\mathbf{Y}_{+}\right]\right)^{-1}, \mathbf{C}_{0}^{0}=-\left(U_{1}\left[\mathbf{Y}_{-}\right]\right)^{-1} \cdot U_{1}\left[\mathbf{Y}_{+}\right] \cdot \mathbf{C}_{0}^{1} \\
& \mathbf{C}_{1}^{1}=\left(U_{1}\left[\mathbf{Y}_{+}\right]-U_{1}\left[\mathbf{Y}_{-}\right] \cdot\left(U_{0}\left[\mathbf{Y}_{-}\right]\right)^{-1} \cdot U_{0}\left[\mathbf{Y}_{+}\right]\right)^{-1}, \mathbf{C}_{1}^{0}=-\left(U_{0}\left[\mathbf{Y}_{-}\right]\right)^{-1} \cdot U_{0}\left[\mathbf{Y}_{+}\right] \cdot \mathbf{C}_{1}^{1} \\
& \mathbf{C}_{0}^{1}=-\frac{e^{2 N b}}{2 \kappa N D_{N}}\left(\begin{array}{cc}
\mu_{*}^{-1} N\left[2 \mu_{1}^{-1}-e^{2 N b}\left(2(N b)^{2}+4 \mu_{1}^{-1} N b+2 \mu_{1}^{-2}\right)\right] & -2\left(N \mu_{*}\right)^{-1} e^{2 N b}\left[(N b)^{2}-\left(\mu_{0} \mu_{1}\right)^{-1}\right] \\
-N^{2}\left[1+e^{2 N b}\left(-2(N b)^{2}-2 N b+\kappa \mu_{1}^{-1}+\mu_{0}^{-1}\right)\right] & \kappa+e^{2 N b}\left(2(N b)^{2}-2 \kappa N b+\frac{1+\kappa^{2}}{2}\right)
\end{array}\right) \\
& \mathbf{C}_{0}^{0}=-\frac{1}{2 \kappa N D_{N}}\left(\begin{array}{cc}
2 \mu_{*}^{-1} N\left[-(N b)^{2}+2 \mu_{1}^{-1} N b-\mu_{1}^{-2}+\mu_{1}^{-1} e^{2 N b}\right] & 2\left(N \mu_{*}\right)^{-1}\left[-(N b)^{2}+\left(\mu_{0} \mu_{1}\right)^{-1}\right] \\
N^{2}\left[-2(N b)^{2}+2 N b+\kappa \mu_{1}^{-1}+1+e^{2 N b}\right] & -\left[2(N b)^{2}+2 \kappa N b+\frac{1+\kappa^{2}}{2}+\kappa e^{2 N b}\right]
\end{array}\right)
\end{aligned}
$$

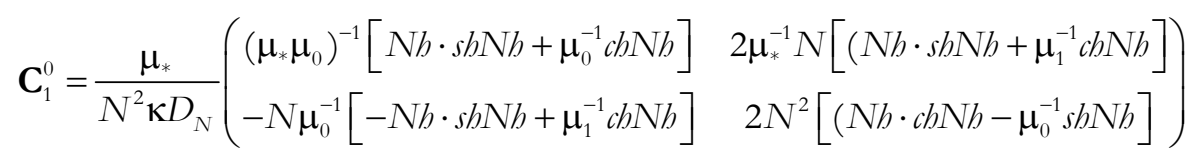

$$
\begin{aligned}
& \mathbf{C}_{1}^{1}=\frac{\mu_{*}}{N^{2} \kappa D_{N}}\left(\begin{array}{cc}
\left(\mu_{*} \mu_{0}\right)^{-1}\left[N h \cdot s h N h+\mu_{0}^{-1} c h N h\right] & 2 \mu_{*}^{-1} N\left[N h \cdot \operatorname{cbNh}+\mu_{1}^{-1} s h N h\right] \\
N \mu_{0}^{-1}\left[-N h \cdot s h N h+\mu_{1}^{-1} c h N h\right] & -2 N^{2}\left[N h \cdot \operatorname{cbNh}-\mu_{0}^{-1} s h N b\right]
\end{array}\right) \\
& D_{N}=2 \operatorname{sh}(2 N h)+4 N h .
\end{aligned}
$$

APPENDIX 4. COMPONENTS OF THE BASIC MATRICES $\Psi_{i}(z), i=0,1$

$$
\begin{aligned}
& \mu_{1}^{-1} \cdot \psi_{0}^{(11)}=2 N^{2} h(b-z) \operatorname{sh} N_{z}-N_{z}\left(1+2 \mu_{1}^{-1}\right) \operatorname{ch} N_{z}+4 \mu_{1}^{-1} N h \cdot \operatorname{ch} N_{z}+ \\
& +2 \mu_{1}^{-2} \operatorname{shNz}+N_{z} \cdot \operatorname{ch} N(2 h-z)+2 \mu_{1}^{-1} \operatorname{shN}(2 b-z) \text {, } \\
& \mu_{1}^{-1} \cdot \psi_{0}^{(12)}=2 h(b-z) \operatorname{shN} z+\kappa N^{-1} z \cdot \operatorname{ch} N z-2\left(\mu_{0} \mu_{1}\right)^{-1} N^{-2} \cdot \operatorname{shN} z+N^{-1} z \cdot \operatorname{ch} N(2 h-z),
\end{aligned}
$$




$$
\begin{aligned}
& \mu_{1}^{-1} \cdot \psi_{0}^{(21)}=N\left[-2 N^{2} b(b-z) c b N_{z}+N_{z}\left(1+2 \mu_{1}^{-1}\right) s h N_{z}-2 N b \cdot s h N_{z}+\right. \\
& \left.+\left(\kappa \mu_{1}^{-1}+\mu_{0}^{-1}\right) c h N z+N z \cdot \operatorname{sh} N(2 h-z)+\operatorname{cb} N(2 h-z)\right] \text {, } \\
& \mu_{1}^{-1} \cdot \psi_{0}^{(22)}=-2 N h(b-z) \operatorname{cb} N z-\kappa(2 h-z) \operatorname{sb} N z-N^{-1} \frac{1+\kappa^{2}}{2} \cdot \operatorname{cb} N z+z \cdot \operatorname{sh} N(2 b-z)-\kappa N^{-1} \operatorname{cb} N(2 h-z), \\
& \psi_{1}^{(11)}=\kappa\left(\mu_{*} \mu_{0}\right)^{-1} N^{-2}\left[N(b-z) c h N(b+z)-N(b+z) \operatorname{cb} N(b-z)+\mu_{0}^{-1}(\operatorname{sh} N(b+z)-\operatorname{sh} N(b-z))\right] \\
& \psi_{1}^{(12)}=2 \kappa \mu_{*}^{-1}\left[-N(b+z) \operatorname{sh} N(b-z)+N(b-z) \operatorname{sh} N(b+z)+\mu_{1}^{-1}(\operatorname{cb} N(b+z)-\operatorname{cb} N(b-z))\right], \\
& \psi_{1}^{(21)}=2 \kappa \mu_{*}^{-1}\left[-N(b-z) s h N(b+z)-N(b+z) s h N(b-z)+\mu_{1}^{-1}(c b N(b+z)+c h N(b-z))\right], \\
& \psi_{1}^{(22)}=4 \kappa \mu_{*}^{-1} \mu_{0} N^{2}\left[-N(b-z) \operatorname{cb} N(b+z)-N(b+z) \operatorname{cb} N(b-z)+\mu_{0}^{-1}(\operatorname{sh} N(b+z)+\operatorname{sh} N(b-z))\right] .
\end{aligned}
$$

\section{APPENDIX 5. FUNCTIONS $F_{1}, F_{2}$ IN THE SOLUTION OF THE THERMOELASTICITY PROBLEM}

$$
\begin{aligned}
& F_{1}=f_{1}+\frac{f_{2}}{2 D_{t}}, \quad F_{2}=f_{3}+\frac{f_{4}+f_{5}+f_{6}+f_{7}}{2 D_{t}}, \\
& f_{1}=e^{-t|z-\xi|} t\left\{t(z-\xi) s h(t \xi)+\mu_{*}^{-1} t|z-\xi| c h(t \xi)-\mu_{*}^{-1} \kappa c h(t \xi)\right\}, \quad f_{2}=f_{21} \cdot \operatorname{sh}(t \xi)-f_{22} \cdot \operatorname{ch}(t \xi), \\
& f_{21}=-e^{-t \xi}\{t \xi \cdot(-2 t(1-z) \operatorname{shtz}+\kappa \operatorname{chtz}+\operatorname{cht}(2-z))- \\
& \left.-2 t^{2}(1-z) c b t z+\kappa t z s h t z+2\left(\mu_{1} \mu_{0}\right)^{-1} \operatorname{cbtz}+t z s h t(2-z)\right\}+ \\
& +e^{t \xi}\left\{t(1-\xi) \cdot\left(-2 t c h t z+2 t z s h t z+\kappa \operatorname{cbtz}+\frac{1}{2} e^{-t(2+z)}+\frac{1}{2} e^{-t(2-z)}\right)-\right. \\
& \left.-\kappa t c h t z+\kappa t z s h t z+2\left(\mu_{1} \mu_{0}\right)^{-1} \operatorname{cbt} z-\frac{1}{2} t(1-z) e^{-t(2+z)}-\frac{1}{2} t(1+z) e^{-t(2-z)}\right\}, \\
& f_{22}=-\mu_{*}^{-1} e^{-t \xi}\{t \xi \cdot(-2 t(1-z) \operatorname{sht} z+\kappa \operatorname{cht} t z+\operatorname{cbt} t(2-z))- \\
& \left.-2 t^{2}(1-z) \operatorname{cbt} z+\kappa t(2-z) \operatorname{sbt} z-\frac{1+\kappa^{2}}{2} \operatorname{cbt} t z+t z \operatorname{sht}(2-z)-\kappa \operatorname{cbt}(2-z)\right\}+ \\
& +\mu_{*}^{-1} e^{t \xi}\left\{-t(1-\xi) \cdot\left(-2 t c h t z+2 t z s h t z+\kappa \operatorname{cb} t z+\frac{1}{2} e^{-t(2+z)}+\frac{1}{2} e^{-t(2-z)}\right)-\right. \\
& \left.-\kappa t c b t z+\kappa t z s h t z+\frac{1+\kappa^{2}}{2} \operatorname{chtz}+\frac{1}{2} t(1-z) e^{-t(2+z)}+\frac{1}{2} t(1+z) e^{-t(2-z)}\right\}, \\
& f_{3}=e^{-t \mid z-\xi}\left\{-2 \mu_{0}^{-1} \operatorname{sgn}(z-\xi) \operatorname{sh}(t \xi)-\mu_{*}^{-1} t|z-\xi| c h(t \xi)+\mu_{*}^{-1} c h(t \xi)-t(z-\xi) s h(t \xi)\right\}, \\
& f_{4}=\kappa \mu_{1} e^{2 t \xi}\left\{t(1-\xi) \cdot\left(2(t-1+\kappa) c h t z-2 t z s h t z-\frac{1}{2} e^{-t(2+z)}-\frac{1}{2} e^{-t(2-z)}\right)+\right. \\
& +(t-1+\kappa) c h t z-t z s h t z+t(1-z) \frac{1}{2} e^{-t(2+z)}+t(1+z) \frac{1}{2} e^{-t(2-z)}- \\
& \left.-\mu_{0}^{-1} \frac{1}{2} e^{-t(2+z)}-\mu_{0}^{-1} \frac{1}{2} e^{-t(2-z)}\right\} \text {, }
\end{aligned}
$$




$$
\begin{aligned}
& f_{5}=\kappa \mu_{1} e^{-2 t \xi}\{t \xi \cdot((\kappa-2) c b t z+2 t(1-z) \operatorname{sht} z-\operatorname{cbt}(2-z))- \\
& -2 t s h t z+2 t^{2}(1-z) \operatorname{cbtz}+\operatorname{cbt} z+t z s h t z-2 \mu_{1}^{-1} t s h t z+ \\
& \left.+t z s h t(2-z)-\mu_{1}^{-2} \operatorname{cbt} z+\mu_{0}^{-1} \operatorname{cbt}(2-z)+2 \mu_{0}^{-2}\left(\kappa \mu_{1}\right)^{-1} \operatorname{cbt} z-\kappa^{-1} \mu_{1}^{-2} \operatorname{cbt} z\right\} \text {, } \\
& f_{6}=-\mu_{1} t \xi \cdot((\kappa-2) \operatorname{cht} z+2 t(1-z) \operatorname{sht} z-\operatorname{cbt}(2-z))+ \\
& +2 \mu_{1} t s h t z-2 \mu_{1} t^{2}(1-z) c h t z+\kappa^{2} \mu_{1} c b t z+\kappa^{2} \mu_{1} t s h t z-\mu_{1}^{-1} c b t z- \\
& -\mu_{1} \operatorname{cbt}(2-z)+\mu_{1} t z \operatorname{sg} t(2-z)-2 \kappa t \operatorname{sb} t z+\kappa \operatorname{cht} t(2-z), \\
& f_{7}=-\mu_{1} t(1-\xi) \cdot\left((2 t-2+\kappa) c h t z-2 t z s h t z-\frac{1}{2} e^{-t(2+z)}-\frac{1}{2} e^{-t(2-z)}\right)- \\
& -\kappa^{2} \mu_{1}(t-1) c b t z+\kappa^{2} \mu_{1} t z s h t z-\mu_{1} t(1-z) \frac{1}{2} e^{-t(2+z)}+\mu_{1} t(1+z) \frac{1}{2} e^{-t(2-z)}- \\
& \left.-\mu_{1} \frac{1}{2} e^{-t(2+z)}-\mu_{1} \frac{1}{2} e^{-t(2-z)}-\frac{\kappa^{2}+1}{2} \operatorname{cbt} z+\kappa \operatorname{cbt} t(2-z)\right\} \text {. }
\end{aligned}
$$

